\author{
ACTA MYCOLOGICA \\ Vol. 44 (1): 77-95 \\ 2009
}

\title{
The participation of macromycetes in selected forest communities of the Masurian Landscape Park (NE Poland)
}

\author{
GRZEGORZ FIEDOROWICZ \\ Department of Mycology, University of Warmia and Mazury in Olsztyn \\ Oczapowskiego 1A, PL-10-719 Olsztyn-Kortowo, grzegorz.fiedorowicz@uwm.edu.pl
}

Fiedorowicz G.: The participation of macromycetes in selected forest communities of the Masurian Landscape Park (NE Poland). Acta Mycol. 44 (1): 77-95, 2009.

Results of mycosociological studies in selected forests communities of the Masurian Landscape Park between 1997 and 2000 are discussed. Observations were conducted in 8 permanent plots and 69 supplementary plots $\left(400 \mathrm{~m}^{2}\right)$. Five plant associations characteristic of the Masurian Landscape Park, Peucedano-Pinetum, Serratulo-Pinetum, Vaccinio uliginosiPinetum, Tilio-Carpinetum and Fraxino-Alnetum, were examined. A total of 335 macromycete species were recorded. The greatest number of species was observed in Tilio-Carpinetum (198).

Key words: macromycetes, Ascomycetes, Basidiomycetes, forest communities, Masurian Landscape Park

\section{INTRODUCTION}

The Masurian Landscape Park (MLP) was established in December 1977 in order to preserve and protect outstanding values of the natural environment of the Masurian Lake District. It aims to protect the richness of the fauna and the flora as well as the cultural and historical heritage. The Park is of great research and teaching importance and is an interesting tourist and recreation area (Dąbrowski, Polakowski and Wołos 1999).

A comparatively clean environment, climatic conditions and the occurrence of a great variety of plant communities relatively unaffected by anthropogenic influence have contributed to the high diversity of fungi and the preservation of many fungal species threatened in Poland or in Europe.

First reports on macromycetes from the area of the former German administrative districts of Sensburg (Mragowo) and Johonnisburg (Pisz) date back to the early $20^{\text {th }}$ century (Abromeit 1905; Neuhoff 1933). Alina Skirgiełło conducted wide- 
ranging mycological observations in the vicinity of Kamien and Ruciane, that is in the area of the MLP at present, in the 1950s. Participants of the IV Congress of European Mycologists collected fungi in the vicinity of Mikołajki, Kamien and Ruciane in September 1966 (Skirgiełło 1968; Kotlaba, Lazebniček 1967). Studies on selected groups of macromycetes in the MLP have also been conducted by Orłoś and Dominik (1960), Domański (1963), Durska (1971), Olesiński and Wojewoda (1985).

A total of 289 taxa of macromycetes have been reported from the MLP in the available literature. No data, however, are given on the phytosociological status of their localities.

\section{STUDY AREA}

The Masurian Landscape Park stretches between longitudes $21^{\circ} 20^{\prime}$ and $21^{\circ} 53^{\prime} \mathrm{E}$ and latitudes $53^{\circ} 36^{\prime}$ and $53^{\circ} 51^{\prime} \mathrm{N}$. The surface area of the MLP is 53.655 ha and that of its protection zone is 18.608 ha. Forests cover $50 \%$ of the area (27.140 ha), waters comprise almost 30\% (15.995 ha) and arable lands constitute nearly 16.6\% (8.920 ha) (Polakowski, Jutrzenka-Trzebiatowski and Hołdyński 1997).

The MLP is situated on the border of three mesoregions in the physiogeographical regionalisation of Poland. The western part of the Park constitutes a fragment of the Pojezierze Mrągowskie Lake District, the eastern part belongs to the Kraina Wielkich Jezior Mazurskich Lake District, and its southern edges are part of the Równina Mazurska Plain (Kondracki 1998).

The occurrence of numerous lakes is a feature that distinguishes the MLP from other protected areas. Those are mostly large lakes whose surface area exceeds 50 ha. They comprise 22 lakes such as Śniardwy, Bełdany, Mokre or Łuknajno. A total of 60 lakes whose surface area is greater than 1 ha have been recorded in the MLP (Bajkiewicz-Grabowska 1989).

The phytosociological composition of the MLP is greatly diversified. Polakowski et al. (1976) distinguished 67 phytocoenoses in the rank of association or community based on their studies. Peucedano-Pinetum and Serratulo-Pinetum dominate among forest communities in the MLP (Polakowski et al. 1997).

\section{MATERIAL AND METHODS}

Mycosociological field studies were carried out between 1997 and 2000. Three hundred and twelve observations were conducted in 8 permanent plots (from May 1997 to December 1999) and 106 observations in 69 supplementary plots in the plant associations examined (Fig. 1). A total of 418 observations were carried out. Observations at permanent plots were conducted every two or three weeks throughout the calendar year. Observations were also made in supplementary plots established in additional patches of phytocoenoses. Supplementary plots were observed between one and eight 


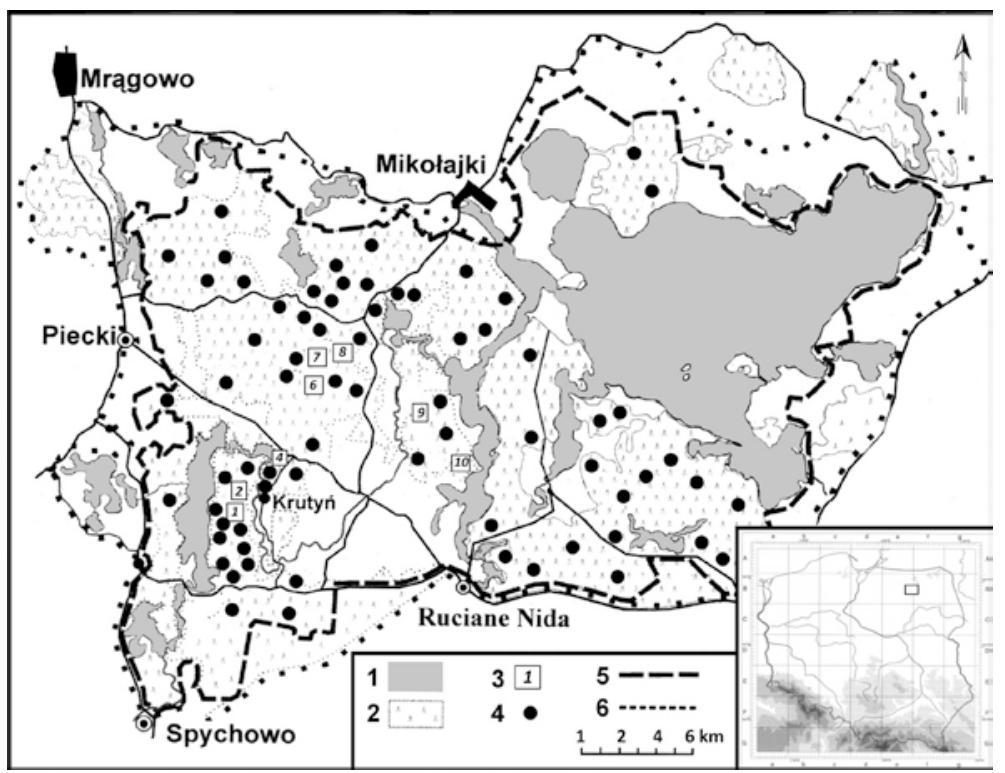

Fig. 1. Location of permanent plots in the Masurian Landscape Park.

1 - waters, 2 - forests, 3 - permanent research plots, 4 - complementary research plots, 5 - boundary of the Park, 6 - boundary of protection zone.

times. The surface area of all observation plots was $400 \mathrm{~m}^{2}$ (Lisiewska 1965; Friedrich 2000). The commonly used Braun-Blanquet scale adjusted for fungi by Moser was used in the quantitative assessment (Nespiak 1959; Lisiewska 1965). The following bioecological groups of fungi were distinguished based on the type and manner of substrate utilization: mycorrhizal fungi, fungi growing on humus, litter-inhabiting fungi, fungi on wood, bryophilous fungi and parasitic fungi (Lisiewska 2000).

The nomenclature of Ascomycetes follows Chmiel (2006) and Hansen \& Knudsen (2000). The nomenclature of Basidiomycetes follows Wojewoda (2003). Names of vascular plants are given according to Mirek et al. (2002) and bryophytes according to Ochyra et al. (2003). Names of phytosociological units are admitted after Matuszkiewicz (2001).

The herbarium material is deposited in the Herbarium of the Chair of Mycology, University of Warmia and Mazury, Olsztyn.

\section{RESULTS AND DISSCUSION}

Mycological observations were conducted in 8 permanent plots. They represented five plant communities: Peucedano-Pinetum (W. Mat. 1962) W. Mat. \& J. Mat. 1973, Vaccinio uliginosi-Pinetum Kleist 1929, Serratulo-Pinetum (W. Mat. 1981) J. Mat. 1988, Tilio-Carpinetum Tracz. 1962, Fraxino-Alnetum W. Mat. 1952 (Matuszkiewicz 2001) (Tab. 1). 
Table 1

Floristic-phytosociological differentiation of forest communities of the Masurian Landscape Park

\begin{tabular}{|c|c|c|c|c|c|c|c|c|}
\hline Successive no. & 1 & 2 & 3 & 4 & 5 & 6 & 7 & 8 \\
\hline Forest community & P.-P. & V.u. $-P$. & S. $-P$. & S. $-P$. & T.-C. & T.-C. & T.-C. & Fr. Al. \\
\hline No. of the permanent research plot & 1 & 2 & 6 & 9 & 4 & 8 & 10 & 7 \\
\hline Forest complex & $\begin{array}{c}\text { Kru- } \\
\text { tyń }\end{array}$ & $\begin{array}{c}\text { Kru- } \\
\text { tyń }\end{array}$ & $\begin{array}{l}\text { Ko- } \\
\text { toin }\end{array}$ & $\begin{array}{l}\text { Gą- } \\
\text { sior }\end{array}$ & $\begin{array}{c}\text { Kru- } \\
\text { tyń }\end{array}$ & $\begin{array}{l}\text { Ko- } \\
\text { toin }\end{array}$ & $\begin{array}{l}\text { Gą- } \\
\text { sior }\end{array}$ & $\begin{array}{l}\text { Ko- } \\
\text { toin }\end{array}$ \\
\hline Forest section & $101 \mathrm{j}$ & $101 \mathrm{c}$ & $245 \mathrm{i}$ & $203 \mathrm{f}$ & $53 \mathrm{f}$ & $221 \mathrm{i}$ & $215 b$ & $221 \mathrm{~g}$ \\
\hline Date & $\begin{array}{l}2.06 . \\
1997\end{array}$ & \begin{tabular}{|l|}
2.06. \\
1997
\end{tabular} & $\begin{array}{l}5.08 \\
1997\end{array}$ & $\begin{array}{l}5.08 \\
1997\end{array}$ & $\begin{array}{l}2.06 . \\
1997\end{array}$ & $\begin{array}{l}2.06 . \\
1997\end{array}$ & $\begin{array}{l}2.06 . \\
1997\end{array}$ & $\begin{array}{l}2.06 \\
1997\end{array}$ \\
\hline Density of tree layer $-\mathrm{a}(\%)$ & 70 & 45 & 80 & 90 & 90 & 80 & 90 & 70 \\
\hline Density of skrub layer - b (\%) & 20 & 10 & 5 & 50 & 5 & 5 & 5 & 5 \\
\hline Cover of herb layer $-\mathrm{c}(\%)$ & 90 & 80 & 30 & 70 & 90 & 80 & 80 & 100 \\
\hline Cover of moss layer $-\mathrm{d}(\%)$ & 90 & 95 & 90 & 10 & 10 & 5 & 5 & 10 \\
\hline Surface of investigated plots in $\mathrm{m}^{2}$ & 400 & 400 & 400 & 400 & 400 & 400 & 400 & 400 \\
\hline Number of plant species & 26 & 23 & 30 & 44 & 34 & 35 & 30 & 27 \\
\hline \multicolumn{9}{|l|}{ Ch. Vaccinio-Piceetea, Dicrano-Pinion } \\
\hline Pinus sylvestris L. & 4.4 & 2.2 & 1.1 & 4.4 & & . & 1.1 & \\
\hline Pinus sylvestris L. & & 1.1 & & & . &. & & \\
\hline Pinus sylvestris L. & + & + & + & + & . & . & 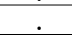 & . \\
\hline Picea abies (L.) H. Karst. & 1.1 & & 4.4 & 1.1 & & & & \\
\hline Picea abies (L.) H. Karst & 1.1 & & + & & . & . & . & \\
\hline Picea abies (L.) H. Karst & + & + & + & + & . & . & . & . \\
\hline Vaccinium myrtillus L. & 4.5 & 1.2 & 1.1 & 1.1 & 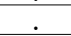 & & & \\
\hline Pleurozium schreberi (Willd. ex Brid) Mitt. d & 4.4 & +.2 & 4.4 & 1.1 & . & . & . & . \\
\hline Ptilium crista-castrensis (Hedw.) De Not. $\mathrm{d}$ & 1.2 & & 2.2 & & & & & \\
\hline Hylocomium splendens (Hedw.) Schimp. & 1.2 & . & 1.2 & + & 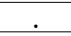 & . & . & . \\
\hline Dicranum polysetum Sw.ex anon. & 1.2 & & & & & & & \\
\hline Trientalis europaea $\mathrm{L}$. & + & & 1.2 & + & 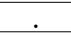 & 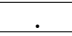 & . & . \\
\hline Vaccinium vitis-idaea $\mathrm{L}$. & 1.2 & + & + & & 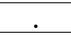 & . &. & $\dot{0}$ \\
\hline Lycopodium annotinum L. & & + & & & & & & \\
\hline Monotropa hypopitys L.s. S. & & & & & + & & . & \\
\hline \multicolumn{9}{|l|}{ Ch. Oxycocco-Sphagnetea } \\
\hline Sphagnum magellanicum Brid. & . & 4.4 & & . & . & . & . & \\
\hline Eriophorum vaginatum L. & & 2.3 & & & $\cdot$ & & & \\
\hline Polytrichum strictum Menzies ex Brid. & & 1.2 & & & . & & 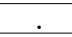 & \\
\hline Sphagnum capillifolium (Ehrh.) Hedw. & & 1.1 & & & $\cdot$ & & & \\
\hline Oxycoccus palustris Pers. & & 1.2 & & . & . & 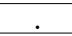 &. & \\
\hline Andromeda polifolia L. & & + & & & & & & \\
\hline Drosera rotundifolia L. & & + & & & & & & \\
\hline \multicolumn{9}{|l|}{ Ch. et D. Peucedano-Pinetum } \\
\hline Peиcedanum oreoselinum (L.) Moench & + & & . & . & $\therefore$ & . & . & \\
\hline \multicolumn{9}{|l|}{ Ch. et D. Vaccinio uliginosi-Pinetum } \\
\hline Vaccinium uliginosum $\mathrm{L}$. & & 3.3 & & 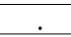 & & & & \\
\hline Ledum palustre $\mathrm{L}$. & & 2.3 & & & & & & \\
\hline \multicolumn{9}{|l|}{ Ch. Querco-Fagetea, Fagetalia sylvaticae } \\
\hline Acer platanoides L. & . & . & 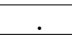 & . & 1.1 & 1.1 & & \\
\hline Acer platanoides L. & $\cdot$ & & & + & + & + & + & \\
\hline Corylus avellana L. & & & 1.1 & 2.2 & + & 1.2 & + & + \\
\hline Dryopteris filix-mas (L.) Schott & . & & + & + & + & + & + & \\
\hline Daphne mezereum L. & & & & + & & + & & \\
\hline Ranunculus lanuginosus $\mathrm{L}$. & 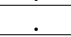 & & & + & & 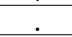 & & + \\
\hline Sanicula europaea L. & & & & + & & & & \\
\hline Anemone nemorosa L. & . & 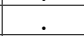 & $\cdot$ & 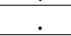 & 2.2 & + & 2.2 & 2.2 \\
\hline Hepatica nobilis Schreb. & & & & & 1.1 & 1.2 & + & \\
\hline Mercurialis perennis $\mathrm{L}$. & 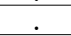 & $\cdot$ & 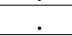 & $\cdot$ & 1.2 & 1.1 & 1.2 & 2.2 \\
\hline Galium odoratum (L.) Scop. & & & & & 1.1 & 1.1 & 1.1 & \\
\hline Galeobdolon luteum Huds. & . & $\cdot$ & . & $\cdot$ & 1.2 & + & + & + \\
\hline Asarum europaeum L. & & & & & 1.2 & + & + & \\
\hline Pulmonaria obscura Dumort. & . & $\cdot$ & . & . & + & 1.1 & + & \\
\hline Lathyrus vernus (L.) Bernh. & & & & & + & + & 1.1 & \\
\hline Poa nemoralis L. & . & . &. & $\cdot$ & + & + & + & \\
\hline Aegopodium podagraria L. & & & & & + & + & + & \\
\hline
\end{tabular}


Tab. 1. cont.

\begin{tabular}{|c|c|c|c|c|c|c|c|c|}
\hline Viola reichenbachiana Jord. ex Boreau & . & . & . & . & + & + & + & \\
\hline Atrichum undulatum (Hedw.) P. Beauv. & & & & & + & + & & \\
\hline Phyteuma spicatum L. & . & $\cdot$ & 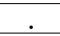 & & + & + & $\cdot$ & \\
\hline Polygonatum multiflorum (L.) All. & & 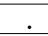 & & . & + & & $\cdot$ & \\
\hline Neottia nidus-avis (L.) Rich. & & & & & + & . & & \\
\hline Lilium martagon $\mathrm{L}$. & . &. & 5 & . & + & & & \\
\hline Lathraea squamaria $\mathrm{L}$. & . & 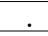 & 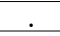 & . & & 1.2 & + & \\
\hline Corydalis solida (L.) Clairv. & & & & & & & 3.3 & \\
\hline Anemone ranunculoides $\mathrm{L}$. & . & $\cdot$ & 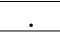 & . & + & . & 1.1 & 3.3 \\
\hline Fraxinus excelsior $\mathrm{L}$. & & . & 5 & & & . & & 3.3 \\
\hline Ulmus glabra Huds. & . & . & . & . & . & . & . & 1.1 \\
\hline Ulmus glabra Huds. & & & & & & & & + \\
\hline Stachys sylvatica $\mathrm{L}$. &. & . & . & . & . & . & & 1.1 \\
\hline Milium effusum L. & & & & & & & & + \\
\hline \multicolumn{9}{|l|}{ Ch. Carpinion betuli } \\
\hline Tilia cordata Mill. & 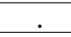 & . & . & . & 4.4 & 3.3 & 2.2 & 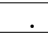 \\
\hline Tilia cordata Mill. & . & . & . & 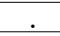 & 1.1 & + & 1.1 & . \\
\hline Tilia cordata Mill. & 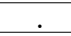 & . & . & . & + & + & + & . \\
\hline Carpinus betulus L. & 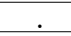 & & . & & 2.2 & 2.2 & 3.3 & \\
\hline Carpinus betulus L. & . & . & . & . & + & & + & . \\
\hline Carpinus betulus L. & $\cdot$ & . & & + & + & + & 1.1 &. \\
\hline Stellaria holostea L. & . & . & + & + & 1.1 & 1.1 & 1.1 & . \\
\hline Dactylis polygama Horv. & 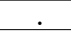 & 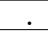 & 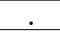 & & + & + & + & \\
\hline \multicolumn{9}{|l|}{ Ch. Alno-Ulmion } \\
\hline $\begin{array}{l}\text { Plagiomnium undulatum (Hedw.) T. J. } \\
\text { Kop. }\end{array}$ & . & . & . & . & + & + & + & 1.1 \\
\hline Gagea lutea (L.) Ker Gawl. & & . & . & . & 1.1 & $\cdot$ & 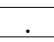 & + \\
\hline Chrysosplenium alternifolium $\mathrm{L}$. & & 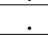 & . & & & . & . & 1.2 \\
\hline Stellaria nemorum L. & & $\cdot$ & & . & & . & . & + \\
\hline Circaea lutetiana $\mathrm{L}$. & & & & & & & & + \\
\hline \multicolumn{9}{|l|}{ Others } \\
\hline Betula pendula Roth. & 1.1 & . & . & & 1.1 & 1.1 & + & \\
\hline Quercus robur L. & & . & . & 2.2 & 1.1 & 1.1 & 1.1 & + \\
\hline Quercus robur L. & 1.1 & & & 2.2 & & & & \\
\hline Quercus robur L. & + & + & + & + & + & . & + & \\
\hline Melampyrum pratense $\mathrm{L}$. & 1.2 & + & 1.1 & + & 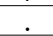 & . & . & \\
\hline Oxalis acetosella $\mathrm{L}$. & + & & 2.2 & 3.3 & & & & \\
\hline Maianthemum bifolium (L.) F.W. Schmidt & + & & + & + & + & . & + & \\
\hline Convallaria majalis $\mathrm{L}$. & & & + & + & & & + & \\
\hline $\begin{array}{l}\text { Plagiomnium affine (Blandow ex Funck) } \\
\text { T. J. Kop. }\end{array}$ & . & & + & + & + & + & + & . \\
\hline Mycelis muralis (L.) Dumort. & . & . & & + & + & + & & + \\
\hline Urtica dioica $\mathrm{L}$. & & & & + & + & + & + & + \\
\hline
\end{tabular}

Sporadic species: Ajuga reptans L. 6 (+); Alnus glutinosa (L.) Gaertn. (a) 8 (2.2); A. glutinosa (L.) Gaertn. (b) 8 (1.1); A. glutinosa (L.) Gaertn. (c) $8(+)$; Anthoxanrhum odoratum L. 1 (+); Athyrium filix-femina (L.) Roth $8(+)$; Betula pendula Roth. (b) $1(+) ;$ B. pendula Roth. (c) $1(+), 3(+)$; B. pubescens Ehrh. (a) 2 (2.2); B. pubescens Ehrh. (b) 2 (1.2); B. pubescens Ehrh. (c) 2 (+); Calamagrostis arundinacea (L.) Roth $1(+), 3(1.1), 4(+) ;$ Calluna vulgaris L. $1(+), 2(+) ;$ Campanula persicifolia L. $6(+) ;$ C. rapunculoides L. $4(+)$; Cardamine flexuosa With. $8(+)$; Carex digitata L. $5(+)$, $7(+)$; Dentaria bulbifera L. 6 (1.1); Deschampsia caespitosa (L.) P. Beauv. $1(+), 3(+), 4(+) ;$ Dicranum scoparium Hedw. (d) $1(+.2), 3$ $(+.2)$; Digitalis grandiflora Mill. 4 (+); Dolichotheca seligeri (Brid) Loeske (d) 3 (1.2), 4 (1.1); Dryopteris carthusiana (Vill.) H. P. Fuchs. $3(+), 4(+)$; Dryopteris cristata (L.) A. Gray $8(+)$; Erodium cicutarium (L.) L'Hér. $6(+)$; Ficaria verna L. 8 (+); Fragaria vesca L. 4 (+); Frangula alnus Mill. (b) 1 (+), 4 (+), 8 (+); Galeopsis tetrahit L. $4(+), 8(+)$; Galium mollugo L. $4(+)$; Geranium robertianum L. $4(+)$; Geum urbanum L. $6(+)$; Glechoma hederacea L. $8(+)$; Hieracium murorum L. $3(+)$; Impatiens parviflora DC. $4(+)$, 8 (+); Leucobryum glaucum (Hedw.) Angstr. (d) 1 (+.2); Luzula pilosa (L.) Willd. 3 (+), 4 (+); Molinia caerulea (L.) Moench 2 (+.2); Polytrichum commune Hedw. (d) 2 (1.2); Polytrichum formosum (Hedw.) G. L. Sm. (d) 1 (+.2), 3 (1.2), 4 (+); Prunella vulgaris L. $6(+)$; Pteridium aquilinum (L.) Kuhn 3 (1.1), 4 (1.1); Rubus ideus L. $3(+), 4$ (1.1); Rumex acetosella L. 1 (+), $3(+), 4(+)$; Sambucus racemosa L. (b) 4 $(+)$; Sorbus aucuparia L. em. Hedl. (c) $1(+), 3(+), 4(+)$; Sphagnum cuspidatum Ehrh. ex Hoffm. (d) 2 (2.2); Stellaria media (L.) Vill. $6(+)$; Veronica officinalis L. $4(+)$; Viola canina L. $4(+)$.

Explanations: P.-P. - Peucedano-Pinetum, V.u.-P. - Vaccinio uliginosi - Pinetum, S.-P. - Serratulo-Pinetum, T.-C. - Tilio-Carpinetum, Fr-Al. - Fraxino-Alnetum. 
Table 2

Macrofungi in the forest communities of the Masurian Landscape Park

\begin{tabular}{|c|c|c|c|c|c|c|c|c|c|c|}
\hline Successive no. & \multicolumn{2}{|c|}{1} & \multirow{2}{*}{\multicolumn{2}{|c|}{2}} & \multicolumn{2}{|c|}{3} & \multicolumn{2}{|c|}{4} & \multicolumn{2}{|c|}{5} \\
\hline Forest community & \multicolumn{2}{|c|}{ P. $P$. } & \multirow{2}{*}{\multicolumn{2}{|c|}{$\frac{\text { V.u. }-P .}{35}$}} & \multirow{2}{*}{\multicolumn{2}{|c|}{$\begin{array}{l}\frac{S .-P .}{173} \\
\end{array}$}} & \multirow{2}{*}{\multicolumn{2}{|c|}{$\begin{array}{c}\text { T.-C. } \\
198\end{array}$}} & \multirow{2}{*}{\multicolumn{2}{|c|}{$\begin{array}{c}F r-A l . \\
66\end{array}$}} \\
\hline Total number of species & \multicolumn{2}{|c|}{72} & & & & & & & & \\
\hline Research plots & $\mathrm{pp}$ & cp & $\mathrm{pp}$ & $\mathrm{cp}$ & $\mathrm{pp}$ & cp & $\mathrm{pp}$ & $\mathrm{cp}$ & $\mathrm{pp}$ & cp \\
\hline Number of plots & 1 & 17 & 1 & 2 & 2 & 25 & 3 & 23 & 1 & 2 \\
\hline Number of observations & 39 & 19 & 39 & 2 & 78 & 31 & 117 & 52 & 39 & 2 \\
\hline Mycorrhiz & al fun & & & & & & & & & \\
\hline Number of species & 49 & & 1 & 9 & 7. & & 5 & 0 & 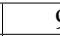 & \\
\hline Paxillus involutus (Batsch: Fr.) Fr. ss. lato & $11^{+-2}$ & $7^{+-2}$ & $9^{+-2}$ & $1^{2}$ & $26^{+-2}$ & $8^{+-2}$ & $38^{+-2}$ & $9^{1-2}$ & $3^{+-1}$ & \\
\hline Xerocomus badius (Fr.: Fr.) Kühner ex Gilbert & $10^{+-1}$ & $8^{+-1}$ & & & $24^{+-3}$ & $3^{1-2}$ & $1^{1}$ & & & \\
\hline Amanita citrina (Schaeff.) Pers. & $7^{+-2}$ & $6^{+-2}$ & & $\cdot$ & $9^{+-2}$ & $2^{+-1}$ & $11^{+-1}$ & $1^{1}$ & & \\
\hline Russula fragilis (Pers.: Fr.) Fr. & & $3^{+-1}$ & & & $2^{1}$ & $1^{1}$ & $5^{+-1}$ & $2^{1}$ & & \\
\hline Cantharellus cibarius Fr. & $8^{+-1}$ & $4^{1}$ & & & $7^{+-2}$ & $4^{1-2}$ & $4^{+-1}$ & $3^{+-1}$ & & \\
\hline Russula vesca Fr. & $4^{+-1}$ & $1^{+}$ & & . & $1^{2}$ & $2^{+-1}$ & $3^{1-2}$ & $3^{+-1}$ & & \\
\hline Russula cyanoxantha (Schaeff.) Fr. & & $1^{1}$ & & & $7^{+-1}$ & $1^{1}$ & $12^{+-1}$ & $1^{1}$ & & . \\
\hline Boletus reticulatus Schaeff. & $2^{+-2}$ & & & & & $2^{1-2}$ & $1^{1}$ & $2^{+-1}$ & & \\
\hline Tricholoma terreum (Schaeff.: Fr.) P. Kumm. ss. lato & & $1^{+}$ & & $\cdot$ & $4^{1}$ & $2^{1}$ & & $2^{+-1}$ & & \\
\hline Lactarius necator (J. F. Gmel.: Fr.) Pers. & $1^{1}$ & $1^{1}$ & & . & $7^{+-2}$ & $3^{+-1}$ & $6^{+-1}$ & $2^{1}$ & . & . \\
\hline Russula velenovskyi Melzer \& Zvára & $3^{+}$ & & & & $2^{+}$ & & $1^{+}$ & & & \\
\hline Amanita fulva (Schaeff.) Pers. & & & $9^{+-2}$ & $1^{+}$ & $4^{+-1}$ & $1^{1}$ & & $2^{+-1}$ & & \\
\hline Lactarius rufus (Scop.: Fr.) Fr. & $15^{+-2}$ & $5^{1-2}$ & $14^{+-4}$ & $2^{1-2}$ & $30^{+-2}$ & $5^{1-3}$ & & &. & . \\
\hline Russula decolorans (Fr.: Fr.) Fr. & $14^{+-2}$ & $3^{1-2}$ & $4^{+-1}$ & $1^{+}$ & $6^{+-1}$ & $3^{+-1}$ & & & & \\
\hline Laccaria proxima (Boud.) Pat. & $8^{+-2}$ & $4^{1-2}$ & $10^{+-1}$ & & $8^{+-2}$ & $1^{1}$ & & & & \\
\hline Suillus variegatus (Schwein.: Fr.) O. Kuntze & $7^{+-2}$ & $1^{1}$ & $6^{+-1}$ & . & & $1^{+}$ & & & & \\
\hline Entoloma cetratum (Fr.: Fr.) M. M. Moser & $6^{+-1}$ & $2^{1}$ & $5^{1-2}$ & & $10^{+-2}$ & $1^{1}$ & & & & \\
\hline Russula vinosa Lindbl. & $7^{+-2}$ & $1^{1}$ & & $1^{+}$ & $7^{+-1}$ & $1^{1}$ & & & & \\
\hline Thelephora terrestris Her. ex Willd.: Fr. & & $1^{1}$ & $6^{+-1}$ & $1^{1}$ & $12^{+-2}$ & $2^{+-1}$ & & . &. &. \\
\hline Leccinum scabrum (Bull.: Fr.) Gray & $2^{1}$ & $1^{+}$ & $1^{+}$ & $1^{1}$ & 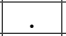 & $2^{1}$ & & & & \\
\hline Lactarius torminosus (Schaeff.: Fr.) Pers. & & $2^{+-1}$ & & $1^{1}$ & & $1^{1}$ & & & & \\
\hline Lactarius vietus (Fr.) Fr. & & $1^{+}$ & & $1^{1}$ & & & &. & $\cdot$ &. \\
\hline Cortinarius semisanguineus (Fr.) Gillet & $14^{+-2}$ & $2^{1-2}$ & & & $5^{+-1}$ & $2^{+-1}$ & & & & \\
\hline Cortinarius armillatus (Fr.: Fr.) Fr. & $9^{+-2}$ & & & & & $1^{1}$ & & & & \\
\hline Inocybe lacera (Fr.: Fr.) P. Kumm. & $9^{+-1}$ & $3^{1}$ & & . & $8^{+-1}$ & $1^{1}$ & & . &. & . \\
\hline Russula emetica (Schaeff.) Pers.: Fr. var. silvestris & $9^{+-1}$ & $4^{+-2}$ & & & $4^{+-1}$ & $6^{+-2}$ & & & & \\
\hline Cortinarius mисоsus (Bull.: Fr.) Kickx & $7^{+-3}$ & $4^{+-2}$ & & . & $3^{+-1}$ & $1^{1}$ & & & & \\
\hline Amanita porphyria (Alb. \& Schwein.: Fr.) Mladý & $6^{+-2}$ & $2^{1}$ & & . & $8^{+-2}$ & $4^{+-2}$ & & . &. & \\
\hline Xerocomus subtomentosus (L.: Fr.) Quél. & $4^{+-1}$ & $2^{+-1}$ & & & $3^{+-1}$ & $2^{1}$ & & & & \\
\hline Tricholoma portentosum (Fr.: Fr.) Quél. & $5^{+-1}$ & $5^{1-2}$ & & . & $3^{+-1}$ & & & & & \\
\hline Tylopilus felleus (Bull.: Fr.) P. Karst. & $5^{+-1}$ & $2^{+-1}$ & & $\dot{.}$ & $9^{+-2}$ & $3^{+-1}$ & & & & \\
\hline Suillus granulatus (L.: Fr.) Roussel & $1^{1}$ & $2^{1-2}$ & & . & $8^{+-2}$ & $2^{1}$ & & . & . & \\
\hline Inocybe dulcamara (Alb. \& Schwein.) P. Kumm. & & $1^{1}$ & & & $6^{+-1}$ & $3^{1}$ & & & & \\
\hline Lactarius mitissimus (Fr.) Fr. & $2^{+-1}$ & $1^{1}$ & & & & $1^{1}$ & & & & \\
\hline Coltricia perennis (L.: Fr.) Murrill & $1^{1}$ & $1^{1}$ & & . & & $2^{1-2}$ & & & . & \\
\hline Suillus luteus (L.: Fr.) Roussel & & $3^{+-2}$ & & & $1^{1}$ & & & & & \\
\hline Russula sanguinea (Bull.) Fr. & & $2^{+-1}$ & & . & & $1^{+}$ & & & & \\
\hline Russula ochroleuca (Pers.) Fr. & & $1^{1}$ & & . & . & $2^{1}$ & & . &. & \\
\hline Russula lutea (Huds.: Fr.) Gray & & $1^{1}$ & & & & $1^{1}$ & & & & \\
\hline Rozites caperatus (Pers.: Fr.) P. Karst. & $5^{1-2}$ & $6^{1-2}$ & & . & & & & & & \\
\hline Boletus pinophilus Pilát \& Dermek & $4^{+-1}$ & $3^{+-1}$ & & . & & & & & & \\
\hline Gomphidius roseus (Fr.) Fr. & $2^{+-1}$ & $2^{+}$ & & & & & & & & \\
\hline Suillus bovinus (L.: Fr.) Roussel & $1^{1}$ & $5^{1-3}$ & & . & & & & & & \\
\hline Leccinum versipelle (Fr.) Snell & $10^{+-2}$ & & & . & & & & & & \\
\hline Cortinarius cinnamomeus $(\mathrm{L} .: \mathrm{F}$ & $7^{+-2}$ & & & & & & & & & \\
\hline Leccinum vulpinum Watling & $1^{+}$ & & & & & & & & & \\
\hline Chalciporus piperatus (Bull.: Fr.) Bat. & & $3^{1-2}$ & & & & & & & & \\
\hline Tricholoma equeste (L.: Fr.) P. Kumm & & $3^{1-2}$ & & & & & & & & \\
\hline Hygrophorus hypothejus (Fr.: Fr.) Fr. & & $1^{1}$ & & & & & & & & \\
\hline Russula versicolor Jul. Schäff. & & $1^{1}$ & & & & & & & & \\
\hline
\end{tabular}


Tab. 2. cont.

\begin{tabular}{|c|c|c|c|c|c|c|c|c|c|c|}
\hline Cortinarius huronensis Ammirati & & & $20^{+-2}$ & $1^{1}$ & & & . & & & \\
\hline Russula emetica (Schaeff.) Pers.: Fr. var. emetica & & & $11^{+-3}$ & $1^{2}$ & & & & & & \\
\hline Leccinum niveum (Fr.) Rauschert & & & $7^{+-2}$ & $1^{+}$ & & & & & & \\
\hline Russula paludosa Britzelm. & & & $6^{+-2}$ & $1^{1}$ & & & & & & \\
\hline Russula betularum Hora & & & $5^{+-1}$ & $1^{+}$ & & & & & & \\
\hline Lactarius helvus (Fr.) Fr. & & & $5^{1-2}$ & $1^{1}$ & & & & & & \\
\hline Lactarius thejogalus (Bull.: Fr.) Gray ss. Neuhoff & & & $3^{+-1}$ & & & & & & & \\
\hline Laccaria laccata (Scop.: Fr.) Berk. \& Broome & & & & . & $9^{+-2}$ & $1^{1}$ & $40^{+-3}$ & $6^{+-2}$ & $2^{1}$ & \\
\hline Inocybe geophylla (Fr.: Fr.) P. Kumm. & & & . & . & $6^{+-2}$ & $1^{1}$ & $18^{+-2}$ & $3^{+-1}$ & $6^{+-1}$ & . \\
\hline Laccaria amethystea (Bull.) Murrill & & & & 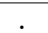 & $14^{+-2}$ & $4^{+-1}$ & $15^{+-2}$ & $4^{1-2}$ & $1^{1}$ & \\
\hline Inocybe fastigiata (Schaeff.) Quél. & & & • & . & $8^{+-2}$ & & $9^{+-2}$ & $4^{+-1}$ & $7^{+-1}$ & \\
\hline Lactarius pyrogalus (Bull.: Fr.) Fr. & . & . & . & . & $6^{+-1}$ & $1^{1}$ & $13^{+-2}$ & $2^{1}$ & $4^{+-1}$ & . \\
\hline Lactarius quietus (Fr.) Fr. & & & & . & $12^{+-1}$ & $1^{1}$ & $23^{+-2}$ & $3^{1-2}$ & & \\
\hline Xerocomus pascuus (Pers.) Krombh. & & & . & . & $10^{+-2}$ & $3^{1}$ & $25^{+-3}$ & $5^{1-3}$ & & . \\
\hline Russula puellaris Fr. & . &. & . & . & $6^{+-1}$ & $1^{1}$ & & $1^{1}$ & . & . \\
\hline Amanita pantherina (DC.: Fr.) Krombh. & & & & & $6^{+-1}$ & $2^{+-1}$ & $3^{+-1}$ & & & \\
\hline Tricholoma sulphureum (Bull.: Fr.) P. Kumm. & & & & & $4^{+-1}$ & $1^{+}$ & $9^{+-1}$ & $2^{1}$ & & \\
\hline Russula pectinata (Bull.) Fr. ss. Romagn. & . & . & . & . & $3^{+-1}$ & & $8^{+-1}$ & $1^{+}$ & . & . \\
\hline Lactarius camphoratus Fr. & & & & - & $2^{1}$ & $1^{1}$ & & $5^{+-2}$ & & \\
\hline Amanita muscaria (L.: Fr.) Hook. & & & & & & $1^{1}$ & $1^{+}$ & $1^{+}$ & & \\
\hline Amanita rubescens (Pers.: Fr.) Gray & . & . & . & . & $1^{1}$ & $3^{+-1}$ & $14^{+-1}$ & $3^{+-1}$ & . & . \\
\hline Craterellus cornucopiodes (L.: Fr.) Pers. & & & . & . & & $1^{2}$ & & $4^{2-4}$ & & 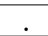 \\
\hline Scleroderma verrucosum (Bull.): Pers. & & & • & . & $2^{+-2}$ & & $1^{+}$ & & & \\
\hline Russula xerampelina (Schaeff.) Fr. & . & . & . & . & $7^{+-1}$ & $2^{+-1}$ & . & . & . & . \\
\hline Boletus edulis Bull.: Fr. & 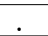 & 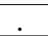 & . & . & $3^{+-1}$ & $4^{+-1}$ & 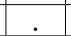 & . & . & . \\
\hline Chroogomphus rutilus (Schaeff.: Fr.) O. K. Miller & & & & & $2^{1}$ & $2^{+-1}$ & 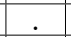 & . & & \\
\hline Russula queletii Fr. & . & . & . & . & $2^{1-2}$ & $1^{+}$ & . & . & . & . \\
\hline Lactarius deterrimus Gröger & 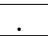 & & . & . & $1^{2}$ & $1^{2}$ & 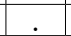 & . & . & \\
\hline Gomphidius glutinosus (Schaeff.: Fr.) Fr. & & & & & $1^{+}$ & $1^{1}$ & 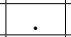 & . & & \\
\hline Russula mustelina Fr. & & & . & . & $5^{+-1}$ & & & $\cdot$ & & \\
\hline Lactarius deliciosus (L.: Fr.) Gray & . & . & . & . & $2^{1}$ & &. & . & . & . \\
\hline Amanita gemmata (Fr.) Bertillon & & & & - & $1^{+}$ & & & & & \\
\hline Lactarius piperatus (L.: Fr.) Gray & & & & . & & $1^{+}$ & $10^{+-1}$ & $3^{+-2}$ & & \\
\hline Gyroporus castaneus (Bull.: Fr.) Quél. & . & & $\cdot$ & . & $\cdot$ & $2^{+-1}$ & $5^{+-1}$ & $1^{+}$ & & \\
\hline Scleroderma citrinum Pers. & & & & 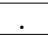 & & $1^{1}$ & $1^{+}$ & $1^{1}$ & & \\
\hline Tricholoma saponaceum (Fr.: Fr.) P. Kumm. & & & & & & $2^{1}$ & & $2^{+-1}$ & & \\
\hline Cortinarius brunneus (Pers.: Fr.) Fr. & . & & $\cdot$ & . & & $2^{+-1}$ & . & . & & . \\
\hline Cortinarius traganus (Fr.: Fr.) Fr. & & & & & & $2^{1}$ & & & & \\
\hline Hygrophoropsis aurantiaca (Wulf.: Fr.) J. Schröt. & & & & & & $2^{1}$ & & $\cdot$ & & \\
\hline Elaphomyces granulatus $\mathrm{Fr}$. & & &. &. & . & $1^{2}$ & . & . & & \\
\hline Lactarius volemus (Fr.) Fr. & & & & & & $1^{+}$ & & & & \\
\hline Russula badia Quél. & & & $\dot{.}$ & . & . & $1^{1}$ & $\dot{0}$ & $\cdot$ & & \\
\hline Russula foetens (Pers.: Fr.) Fr. & . & &. & . & . & $1^{+}$ & . & . & & \\
\hline Suillus grevillei (Klotzsch: Fr.) Singer & & & & & & $1^{2}$ & & & & \\
\hline Hydnum repandum L.: Fr. & & & . & . & 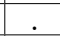 & & $13^{+-2}$ & $6^{+-2}$ & & \\
\hline $\begin{array}{l}\text { Entoloma rhodopolium for. nidorosum (Fr.) } \\
\text { Noordel. }\end{array}$ & . & $\cdot$ & . & . & . & . & $13^{+-1}$ & $4^{1}$ & . & . \\
\hline Tricholoma lascivum (Fr.: Fr.) Gillet & & & & 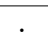 & & & $13^{+-1}$ & $2^{+-1}$ & & \\
\hline Russula nigricans (Bull.: Fr.) Fr. & & & & & & & $10^{1-2}$ & $2^{1}$ & & \\
\hline Russula risigallina (Batsch) Sacc. & & & & . & & & $9^{+-1}$ & $2^{1-2}$ & & \\
\hline Amanita phalloides (Vaill.: Fr.) Link & & & & . & & & $7^{+-1}$ & $5^{+-2}$ & & \\
\hline Hebeloma crustuliniforme (Bull.) Quél. & & & $\cdot$ & 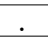 & & & $7^{+-2}$ & $2^{+-1}$ & & \\
\hline Leccinum pseudoscabrum (Kallenb.) Šutara & & & & . & & & $5^{+-2}$ & $8^{+-2}$ & & \\
\hline Lactarius vellereus (Fr.) Fr. & & & & . & & & $3^{+-1}$ & $1^{1}$ & & \\
\hline Russula delica Fr. & & & $\cdot$ & . & & & $8^{+-2}$ & & & \\
\hline Amanita vaginata (Bull.: Fr.) Vittad & & & $\cdot$ & . & & & $1^{1}$ & & & \\
\hline Boletus luridus Schaeff.: Fr. & & & & . & & & & $1^{1}$ & & \\
\hline Cortinarius orellanus Fr. & & & 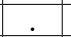 & . & . & & . & $1^{1}$ & & \\
\hline Inocybe erubescens Blytt & & & & 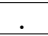 & & & $\dot{0}$ & $1^{1}$ & & \\
\hline Leccinum aurantiacum (Bull.) Gray & & & & & & & & $1^{2}$ & & \\
\hline
\end{tabular}


Tab. 2. cont.

\begin{tabular}{|c|c|c|c|c|c|c|c|c|c|c|}
\hline Pseudocraterellus undulatus (Pers.: Fr.) Rauschert & & & & & & & & $1^{4}$ & & \\
\hline Russula solaris Ferd. \& Winge & & & & & & & & $1^{1}$ & & \\
\hline Xerocomus rubellus (Krombh.) Quél. & & & & . & & & & $1^{1}$ & & \\
\hline Cortinarius paleaceus $\mathrm{Fr}$. & & & & & & & & & $4^{+-1}$ & \\
\hline Lactarius lilacinus (Lasch: Fr.) Fr. & & & & . & & & & & $3^{+-1}$ & \\
\hline Paxillus rubicundulus P. D. Ordon & & & & . & & & & . & $1^{+}$ & \\
\hline \multicolumn{11}{|c|}{ Saprotrophic fungi on humus } \\
\hline Number of species & 0 & & \multicolumn{2}{|c|}{0} & \multicolumn{2}{|c|}{13} & \multicolumn{2}{|c|}{36} & \multicolumn{2}{|c|}{8} \\
\hline Lycoperdon perlatum Pers.: Pers. & & & & & & $1^{1}$ & $20^{+-2}$ & $3^{1}$ & $5^{1-2}$ & \\
\hline Humaria hemisphaerica (F. H. Wigg.: Fr.) Fuckel & & & & & $5^{+-1}$ & & $18^{+-3}$ & $7^{+-2}$ & & \\
\hline Cystolepiota seminuda (Lasch) Bon & & & & . & $3^{+-1}$ & $1^{+}$ & $27^{+-2}$ & $4^{+-1}$ & $5^{+-1}$ & \\
\hline Agaricus silvicola (Vittad.) Peck & & & . & - & $3^{+-1}$ & $1^{+}$ & $4^{+-1}$ & . & & . \\
\hline Lycoperdon umbrinum Pers.: Pers. & & & . & . & $14^{1-2}$ & $4^{+-2}$ & & . & & \\
\hline Otidea leporina (Batsch: Fr.) Fuckel & . & & $\dot{ }$ & . & $3^{1-2}$ & $1^{2}$ &. & . & . & $\cdot$ \\
\hline Agaricus silvaticus Schaeff. & & & & 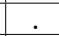 & $3^{+-1}$ & & & & & \\
\hline Peziza badia Pers.: Fr. & & & 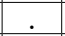 & . & & $3^{+-1}$ & $\dot{.}$ & . & . & . \\
\hline Geoglossum umbratile Sacc. & & & & & & $1^{3}$ & & . & $\cdot$ & \\
\hline Gyromitra esculenta (Pers.) Fr. & . & . & . & . & . & $1^{1}$ & . & . & . & . \\
\hline Lyophyllum decastes (Fr.: Fr.) Singer & & & . & . & & $1^{2}$ & & $\cdot$ & . & \\
\hline Macrolepiota rhacodes (Vittad.) Singer & & & & & & $1^{1}$ & & & & \\
\hline Morchella conica Pers. & . & . & . & . & . & $1^{1}$ & & & & 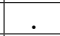 \\
\hline Phallus impudicus L.: Pers. & . & $\cdot$ & . & . & . & & $19^{+-2}$ & $4^{1-2}$ & $2^{+}$ & \\
\hline Lepiota cristata (Bolt.: Fr.) P. Kumm. & & & & & & & $6^{+-1}$ & $4^{1-2}$ & $5^{+-1}$ & \\
\hline Gyromitra gigas (Krombh.) Cooke & . & - & . & . & . & . & $2^{+-1}$ & $2^{1-2}$ & $1^{+}$ & . \\
\hline Clavulina coralloides (L.: Fr.) J. Schröt. & & & & 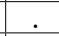 & & & $24^{+-2}$ & $6^{+-1}$ & & \\
\hline Bovista nigrescens Pers.: Pers. & & & & & & & $16^{+-2}$ & & & \\
\hline Macrolepiota procera (Scop.: Fr.) Singer & . & . & . & . & $\cdot$ & & $14^{+-2}$ & $2^{1}$ & . & . \\
\hline Calvatia excipuliformis (Scop.: Pers.) Perdeck & & & & $\cdot$ & & & $10^{+-1}$ & $2^{1}$ & & \\
\hline Clavulina cinerea (Bull.: Fr.) J. Schröt. & & & & & & & $6^{+-1}$ & $2^{1}$ &. & \\
\hline Calocybe gambosa (Fr.) Donk & . & . & . & . & . & . & $3^{+-2}$ & $1^{1}$ & . & . \\
\hline Helvella macropus (Pers.) P. Karst. & & & & . & - & & $3^{+-1}$ & $1^{1}$ & & \\
\hline Lepiota subgracilis Kühner ex Wasser & & & & & & & $3^{+}$ & & & \\
\hline Peziza arvernensis Boud. & . & $\dot{0}$ & . & . & . & . & $3^{+-2}$ & & . & . \\
\hline Otidea onotica (Pers.: Fr.) Fuckel & & - & . & . & 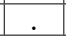 & & $2^{1-2}$ & $3^{2-3}$ & - & . \\
\hline Coprinus xanthothrix Romagn. & & & & & & & $2^{+-1}$ & $1^{1}$ & & \\
\hline Peziza succosa Berk. & . & $\dot{0}$ & . & . & . & . & $2^{1}$ & . & . & . \\
\hline Peziza vesiculosa Bull. & & - & - & . & - & & $2^{+-1}$ & - & & \\
\hline Clavariadelphus pistillaris (L.: Fr.) Donk & & & & . & & & $1^{+}$ & & & \\
\hline Agaricus placomyces Peck & . & $\dot{0}$ & . & . & . & . & $1^{1}$ & $1^{1}$ & . & . \\
\hline Lycoperdon molle Pers. & & 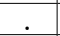 & 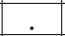 & . & - & 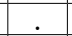 & $1^{+}$ & & & \\
\hline Helvella crispa (Scop.) Fr. & & & & . & & & & $6^{1-2}$ & & \\
\hline Scutellinia scutellata (L.) Lambotte & & & & & & & & $6^{1-2}$ & & \\
\hline Coprinus atramentarius (Bull.: Fr.) Fr. & & & . & . & & & & $2^{1-2}$ & & \\
\hline Helvella elastica Bull. & & & & . & & & & $2^{1}$ & & \\
\hline Thelephora palmata (Scop.): Fr. & & & & & & & & $2^{1}$ & & 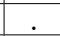 \\
\hline Albatrellus confluens (Fr.) Kotl. \& Pouzar & & & & . & & & & $1^{2}$ & & \\
\hline Aleuria aurantia (Pers.: Fr.) Fuckel & & & & & & & & $1^{4}$ & & \\
\hline Calvatia utriformis (Bull.: Pers.) Jaap & & & & & & & & $1^{1}$ & & $\cdot$ \\
\hline Helvella acetabulum (L.: Fr.) Quél. & & & & . & & & & $1^{2}$ & & \\
\hline Langermannia gigantea (Batsch: Pers.) Rostk. & & & & & & & & $1^{+}$ & & \\
\hline Lyophyllum connatum (Schum.: Fr.) Singer & & . & • &. & • & & . & $1^{2}$ & & \\
\hline Morchella esculenta (L.) Pers. & & & & . & & & & $1^{+}$ & & \\
\hline Otidea cochleata (L.) Fuckel & & & & & & & & $1^{2}$ & & \\
\hline Conocybe tenera (Schaeff.: Fr.) Fayod & & & & • & 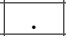 & & $\dot{.}$ & . & $8^{+-2}$ & \\
\hline Ramaria aurea (Schaeff.: Fr.) Quél. & & & & & & & & & $8^{1-2}$ & \\
\hline Mutinus caninus (Huds.: Pers.) Fr. & & & & & & & & & $3^{+-1}$ & \\
\hline \multicolumn{11}{|c|}{ Saprotrophic fungi on litter } \\
\hline Number of species & & & & 4 & & 27 & 21. & 1 & 1. & 11 \\
\hline Mycena galopus (Pers.: Fr.) P. Kumm. & $11^{+-2}$ & $7^{+-2}$ & $13^{+-2}$ & $1^{1}$ & $32^{+-3}$ & $8^{+-2}$ & $9^{+-1}$ & $4^{+-1}$ & $11^{+-2}$ & \\
\hline Setulipes androsaceus (L.: Fr.) Antonín & $13^{1-5}$ & $6^{1-4}$ & $22^{+-4}$ & $2^{1-2}$ & $23^{+-4}$ & $3^{1-2}$ & & & & \\
\hline
\end{tabular}


Tab. 2. cont.

\begin{tabular}{|c|c|c|c|c|c|c|c|c|c|c|}
\hline Myce & $8+2$ & $41-2$ & $10^{+-3}$ & 11 & 7 & 2 & & & & \\
\hline $\begin{array}{l}\text { Rhodocollybia butyracea for. asema (Fr.: Fr.) } \\
\text { Antonín, Halling \& Noordel }\end{array}$ & $\begin{array}{l}0 \\
8^{+-1}\end{array}$ & $44^{1-2}$ & 10 & . & $20^{+-4}$ & $3^{1-2}$ & . & . & & . \\
\hline Cystoderma amianthinum (Scop.: Fr.) Fayod & $7^{+-2}$ & $4^{+-1}$ & & 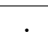 & $11^{+-2}$ & $1^{1}$ & & & & \\
\hline Strobilurus tenacellus (Pers.: Fr.) Singer & & $1^{1}$ & $6^{+-1}$ & . & $10^{1-2}$ & & & & & \\
\hline Gymnopus dryophilus (Bull.: Fr.) Murrill & & $2^{1}$ & & . & $16^{+-2}$ & $1^{2}$ & $38^{+-2}$ & $7^{1-3}$ & $12^{+-2}$ & $1^{1}$ \\
\hline $\begin{array}{l}\text { Mycena sanguinolenta (Alb. \& Schwein.: Fr.) P. } \\
\text { Kumm. }\end{array}$ & . & $1^{2}$ & . & . & $10^{+-1}$ & $3^{1-2}$ & $10^{1-2}$ & $4^{1-2}$ & $3^{1-2}$ & . \\
\hline Auriscalpium vulgare Gray & & $3^{1}$ & . & . & $22^{+-2}$ & $11^{+-2}$ & $3^{1}$ & & & \\
\hline Mycena pura (Pers.: Fr.) P. Kumm. & & & & & $17^{+-2}$ & $4^{1}$ & $33^{+-2}$ & $4^{1-2}$ & $10^{+-2}$ & \\
\hline Mycena vitilis (Fr.) Quél. & & & & . & $6^{+-2}$ & & $40^{+-2}$ & $6^{+-2}$ & $3^{+-1}$ & \\
\hline Clitocybe gibba (Pers.: Fr.) P. Kumm. & & . & . & . & $9^{+-2}$ & $1^{+}$ & $27^{+-2}$ & $2^{1}$ & & \\
\hline Hymenoscyphus fructigenus (Bull.) Fr. & . & . &. & . & $1^{1}$ & & $7^{1-2}$ & $2^{1-2}$ & . & . \\
\hline Psilocybe aeruginosa (M. A. Curtis: Fr.) Noordel. & & & & & & $2^{1}$ & $21^{+-1}$ & $1^{2}$ & & \\
\hline Mycena stylobates (Pers.: Fr.) P. Kumm. & & . & . & . & $6^{1-2}$ & & $18^{+-2}$ & $5^{1-2}$ & & . \\
\hline $\begin{array}{l}\text { Gymnopus confluens (Pers.: Fr.) Antonín, Halling } \\
\text { \& Noordel. }\end{array}$ & . & . & $\cdot$ & . & $5^{1-2}$ & $1^{2}$ & $3^{2}$ & $1^{3}$ & . & . \\
\hline $\begin{array}{l}\text { Gymnopus peronatus (Bolt.: Fr.) Antonín, Halling } \\
\text { \& Noordel. }\end{array}$ & . & . & . & . & $4^{+-2}$ & $2^{+-3}$ & $3^{1}$ & . & & . \\
\hline Clitocybe clavipes (Pers.: Fr.) P. Kumm. & & . & & . & $19^{+-2}$ & $4^{+-1}$ & & . & & \\
\hline Mycena zephirus (Fr.: Fr.) P. Kumm. & & . &. & . & $9^{+-2}$ & $4^{1-2}$ & . &. & & . \\
\hline Strobilurus esculentus (Wulf.: Fr.) Singer & & & & & $8^{+-4}$ & & & & & \\
\hline Mycena aetitis (Fr.) Quél. & & . & & . & $7^{+-1}$ & & & . & & \\
\hline Ramaria abietina (Pers.: Fr.) Quél. & & . & & $\dot{0}$ & $6^{1-2}$ & $1^{1}$ & . &. & & \\
\hline Marasmius scorodonius (Fr.: Fr.) Fr. & & & & & $2^{+-2}$ & $1^{2}$ & & $\dot{.}$ & & \\
\hline $\begin{array}{l}\text { Marasmiellus perforans (Hoffm.: Fr.) Antonín, } \\
\text { Halling \& Noordel. }\end{array}$ & . & . & . & . & $2^{1-2}$ & $1^{2}$ & . & & & \\
\hline Rutstroemia bulgarioides (Rabenh.) P. Karst. & & & & & & $1^{2}$ & & & & \\
\hline Strobilurus stephanocytis (Hora) Singer & &. & . & . & . & $1^{1}$ & . &. & & \\
\hline Cystodermella granulosum (Batsch: Fr.) Harmaja & & & & & & $1^{1}$ & & & & \\
\hline Marasmius rotula (Scop.: Fr.) Fr. & . & . &. & . & . & . & $44^{+-4}$ & $9^{1-3}$ & $18^{1-4}$ & $\cdot$ \\
\hline Rhodocollybia butyracea (Bull.: Fr.) Lennox & & & & . & & . & $26^{+-2}$ & $6^{+-2}$ & $2^{1}$ & \\
\hline Clitocybe nebularis (Batsch: Fr.) P. Kumm. & & & & & & . & $8^{+-2}$ & $3^{1}$ & $6^{+-1}$ & \\
\hline Tubaria furfuracea (Pers.: Fr.) Gillet & . & . & . & 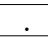 & . & . & $9^{+-3}$ & $3^{1-2}$ & $4^{1-2}$ & . \\
\hline Mycena polyadelpha (Lasch) Kühner & & $\dot{.}$ & . &. & . & . & $13^{1-4}$ & $5^{1-3}$ & & \\
\hline $\begin{array}{l}\text { Marasmiellus foetidus (Sowerby: Fr.) Antonín, } \\
\text { Halling \& Noordel. }\end{array}$ & . & . & . & $\cdot$ & . & . & . & $1^{4}$ & . & . \\
\hline Clitocybe geotropa (Bull.) Quél. & & . & . & . & & & $5^{+-1}$ & $2^{1}$ & & \\
\hline Setulipess quercophilus (Pouzar) Antonín & & $\dot{5}$ & &. & . & . & $6^{1-2}$ & $2^{2}$ & & $\cdot$ \\
\hline Lepista nuda (Bull.: Fr.) Cooke & & $\dot{.}$ & &. & 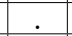 & . & . & $2^{1}$ & & \\
\hline $\begin{array}{l}\text { Hymenoscyphus albidus (Roberge ex Desm.) } \\
\text { W. Phillips }\end{array}$ & . & . & • & . & . & . & & & $13^{3-5}$ & . \\
\hline Mycena rubromarginata (Fr.: Fr.) P. Kumm. & & & & & & & & & $3^{1-2}$ & \\
\hline \multicolumn{11}{|c|}{ Saprotrophic fungi on wood } \\
\hline Number of species & \multicolumn{2}{|c|}{10} & \multicolumn{2}{|c|}{4} & \multicolumn{2}{|c|}{41} & \multicolumn{2}{|c|}{76} & \multicolumn{2}{|c|}{30} \\
\hline Stereum hirsutum (Willd.: Fr.) Gray & $\mathrm{x}^{2}$ & $1^{1}$ & & & $\mathrm{x}^{2}$ & $8^{1-2}$ & $3 \mathrm{x}^{2}$ & $27^{1-3}$ & $\mathrm{x}^{3}$ & $1^{2}$ \\
\hline Exidia plana (Wiggers) Donk & $11^{1-2}$ & $2^{2}$ & & & $12^{1-2}$ & $4^{2}$ & $35^{1-3}$ & $10^{2-3}$ & & . \\
\hline Pluteus atricapillus (Batsch) Fayod & & & $1^{+}$ & 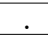 & $5^{+-1}$ & $4^{+-1}$ & $16^{+-2}$ & $3^{+-1}$ & $4^{+-1}$ & \\
\hline Piptoporus betulinus (Bull.: Fr.) P. Karst. & & & $5^{1}$ & & & & $10^{+-1}$ & $3^{1}$ & & \\
\hline Polyporus brumalis (Pers.): Fr. & & $1^{+}$ &. & . & & $1^{+}$ & $3^{1}$ & . & & . \\
\hline Calocera viscosa (Pers.: Fr.) Fr. & $5^{+-1}$ & $1^{1}$ & & & $5^{+-1}$ & $3^{1-2}$ & & & & \\
\hline $\begin{array}{l}\text { Xeromphalia campanella (Batsch: Fr.) Kühner \& } \\
\text { Maire }\end{array}$ & $5^{1-3}$ & $1^{2}$ & . & . & . & $4^{1-4}$ & . & . & & . \\
\hline Psilocybe capnoides (Fr.: Fr.) Noordel. & $2^{1-2}$ & $2^{1-4}$ & & & $4^{2}$ & $3^{2-3}$ & $\dot{\theta}$ & . & & \\
\hline Gymnopilus penetrans (Fr.: Fr.) Murrill & & $2^{1}$ & . & . & $7^{1-2}$ & $3^{1-2}$ & . &. & & \\
\hline Lentinus lepideus (Fr.: Fr.) Fr. & & $1^{2}$ & & & $1^{2}$ & & $\cdot$ & & & \\
\hline Trichaptum abietinum (Dicks.: Fr.) Ryvarden & & $3^{1-2}$ & & $1^{1}$ & $\mathrm{x}^{2}$ & $1^{2}$ & & & & \\
\hline $\begin{array}{l}\text { Trichaptum fuscoviolaceum (Ehrenb.: Fr.) } \\
\text { Ryvarden }\end{array}$ & . & $1^{2}$ & . & . & $2 x^{2}$ & $4^{1-2}$ & . & . & & . \\
\hline $\begin{array}{l}\text { Rhodocollybia maculata (Alb. \& Schwein.: Fr.) } \\
\text { Singer }\end{array}$ & & . & $1^{1}$ & . & . & . & . & . & & \\
\hline
\end{tabular}


Tab. 2. cont.

\begin{tabular}{|c|c|c|c|c|c|c|c|c|c|c|}
\hline Stereum rugosum (Pers.: Fr.) Fr. & & & & & $\mathrm{x}^{1-3}$ & & $2 x^{1-3}$ & $8^{2}$ & $\mathrm{x}^{3}$ & $1^{2}$ \\
\hline Mycena galericulata (Scop.: Fr.) Gray & & & & & $5^{+-1}$ & $1^{2}$ & $32^{+-2}$ & $3^{1-2}$ & $14^{1-2}$ & \\
\hline Psilocybe lateritia (Schaeff.: Fr.) Noordel. & & & & & $1^{2}$ & & $1^{2}$ & $1^{1}$ & $1^{2}$ & . \\
\hline Psilocybe fasticularis (Huds.: Fr.) Noordel. & & & & & $10^{1-3}$ & $2^{2}$ & $16^{2-4}$ & $6^{2-4}$ & $1^{2}$ & \\
\hline Ramaria stricta (Pers.: Fr.) Quél. & & & & & & $1^{1}$ & $3^{+-1}$ & $3^{1-2}$ & & $1^{1}$ \\
\hline Xylaria hypoxylon (L.) Grev. & & & & & & $4^{1-2}$ & $3 x^{1-4}$ & $28^{2-3}$ & $\mathrm{x}^{3}$ & $1^{2}$ \\
\hline Xylaria polymorpha (Pers.) Grev. & & & & & & $2^{2}$ & $3 x^{1-3}$ & $25^{1-3}$ & $\mathrm{x}^{1}$ & $1^{2}$ \\
\hline Tremella mesenterica Retz.: Fr. & & & & & & $1^{1}$ & $2^{1}$ & & & $\dot{0}$ \\
\hline Daedalea quercina (L.: Fr.) Pers. & & . & . & . & & $1^{1}$ & & $1^{1}$ & . & . \\
\hline Exidia glandulosa (Bull.): Fr. & & & & & $6^{1-2}$ & & $5^{1-2}$ & $3^{1-2}$ & & \\
\hline $\begin{array}{l}\text { Hyphodontia paradoxa (Schrad.: Fr.) E. Langer \& } \\
\text { Vesterholt ss. lato }\end{array}$ & & . & . & . & $\mathrm{x}^{2}$ & . & $3 \mathrm{x}^{3-4}$ & $13^{2-4}$ & . & . \\
\hline Pholiota mutabilis (Scop.: Fr.) P. Kumm. & & & & & $7^{1-2}$ & $2^{1-2}$ & $18^{1-2}$ & $4^{2}$ & & \\
\hline Crepidotus variabilis (Pers.: Fr.) P. Kumm. & & . & . & & & $3^{1-2}$ & $25^{+-2}$ & $3^{1-2}$ & . & . \\
\hline Trametes versicolor (L.: Fr.) Pilát & & . & . & & & $1^{2}$ & $2 \mathrm{x}^{2}$ & $2^{1-2}$ & & \\
\hline Geopyxis carbonaria (Alb. \& Schwein.: Fr.) Sacc. & & . & . & & & $1^{1}$ & & & & \\
\hline Lycoperdon pyriforme Schaeff.: Pers. & & . & . & . & $17^{+-2}$ & $2^{1-2}$ & $18^{+-2}$ & $4^{1-2}$ & . & . \\
\hline Mycena stipata Maas Geest. \& Schwöb. & & & 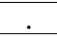 & & $8^{1-2}$ & & $5^{1-2}$ & $3^{1-2}$ & & \\
\hline Stereum sanguinolentum Alb. \& Schwein.: Fr.) Fr. & & & & & $31^{1-3}$ & $2^{2}$ & & & & \\
\hline Pseudohydnum galatinosum (Scop.: Fr.) P. Karst. & & . & . & . & $5^{+-1}$ & . & . & . & . & . \\
\hline Pluteus atromarginatus (Singer) Kühner & & & . & & $3^{+-1}$ & & & & & $\cdot$ \\
\hline Tricholomopsis rutilans (Schaeff.: Fr.) Singer & & & & & $2^{+-1}$ & $1^{1}$ & & & & \\
\hline Discina ancilis (Pers.) Sacc. & & . & . & $\dot{.}$ & 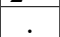 & $1^{2}$ & . &. & . & . \\
\hline $\begin{array}{l}\text { Oligoporus stipticus (Pers.: Fr.) Gilbertson \& } \\
\text { Ryvarden }\end{array}$ & & . & . & . & & $4^{+-1}$ & . & . & . & . \\
\hline Panellus mitis (Pers.: Fr.) Singer & & . & . & & & $3^{2}$ & . & & &. \\
\hline Gloephyllum odoratum (Wulf.: Fr.) Imaz. & & & . & & & $2^{1}$ & &. & & \\
\hline Gloephyllum sepiarum (Wulf.: Fr.) P. Karst. & & . & . & . & . & $1^{1}$ & . & . & . & . \\
\hline Hymenochaete tabacina (Sowerby) Lév. & & & & & & $1^{2}$ & $\cdot$ & & & 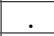 \\
\hline Phaeolus schweinitzii (Fr.: Fr.) Pat. & & . & . & 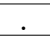 & & $1^{+}$ &. & . & & . \\
\hline $\begin{array}{l}\text { Phlebia tremellosa (Schrad.: Fr.) Nakasone \& } \\
\text { Burds. }\end{array}$ & & . & . & . & & $1^{1}$ & . & . & . & . \\
\hline $\begin{array}{l}\text { Oligoporus caesius (Schrad.: Fr.) Gilbertson \& } \\
\text { Ryvarden }\end{array}$ & & . & . & . & & $1^{1}$ & $\cdot$ & . & . & . \\
\hline Ganoderma applanatum (Pers.) Pat. & & . & . & & & . & $2 x^{1-2}$ & $1^{1}$ & 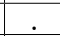 & $1^{1}$ \\
\hline Hymenochaete rubiginosa (Schrad.: Fr.) Lév. & & & . & & & & $\mathrm{x}^{2}$ & $1^{2}$ & & \\
\hline Megacollybia platyphylla (Pers.: Fr.) Kotl. \& Pouzar & & & & & & & $18^{+-2}$ & $5^{+-1}$ & $10^{+-2}$ & \\
\hline Polyporus ciliatus Fr.: Fr. & & . & . & . & . & . & $12^{1-4}$ & $3^{2-3}$ & $6^{1}$ & $1^{2}$ \\
\hline Mycena inclinata (Fr.) Quél. & & & $\cdot$ & & & & $9^{2-4}$ & & $7^{2-5}$ & \\
\hline Panellus serotinus (Schrad.: Fr.) Kühner & & & & & & & $9^{2-3}$ & & $4^{2}$ & \\
\hline Polyporus badius (Pers.) Schwein. & & . & . & 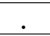 & & $\cdot$ & $7^{2-3}$ & & & $1^{+}$ \\
\hline Sarcoscypha austriaca (O. Beck ex Sacc.) Boud. & & & & & & & $7^{1-4}$ & $4^{1-2}$ & $6^{+-3}$ & $1^{2}$ \\
\hline Delicatula integrella (Pers.: Fr.) Fayod & & & & & & & $2^{2}$ & $1^{2}$ & $2^{1-2}$ & \\
\hline Datronia mollis (Sommerf.: Fr.) Donk & & . & . &. & &. & & $8^{1-2}$ & $x^{2}$ &. \\
\hline Polyporus melanopus (Pers.): Fr. & & & & & & & $1^{1}$ & & $1^{1}$ & \\
\hline Coprinus disseminatus (Pers.: Fr.) Quél. & & & & & & & & $3^{2-3}$ & $2^{3}$ & \\
\hline Polyporus squamosus (Huds.): Fr. & & . & . & & & . & . & $2^{1-2}$ & $5^{1}$ & \\
\hline Bjerkandera adusta (Willd.: Fr.) P. Karst. & & & & & & & & $4^{1-2}$ & & $1^{2}$ \\
\hline Discina parma . Breitenb. \& Maas Geest. & & & & & & & & $1^{2}$ & & $1^{1}$ \\
\hline Trametes hirsuta (Wulf.: Fr.) Pilát & & . & . & & & . & $2 x^{2}$ & $5^{1-2}$ & & . \\
\hline Peniophora quercina (Pers.: Fr.) Cooke & & & & & & & $2 \mathrm{x}^{2-3}$ & $5^{2}$ & & \\
\hline Xylaria longipes Nitschke & & & & & & & $2 x^{1-2}$ & $10^{1-2}$ & & \\
\hline Cylindrobasidium laeve (Pers.: Fr.) Chamuris & & . & . & & & . & $2 \mathrm{x}^{2}$ & $7^{2}$ & &. \\
\hline Nectria cinnabarina (Tode: Fr.) Fr. & & 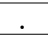 & 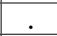 & & & & $\mathrm{x}^{3}$ & $4^{2-3}$ & & . \\
\hline Peniophora incarnata (Pers.: Fr.) P. Karst. & & & & & & & $\mathrm{x}^{2}$ & $4^{2}$ & & \\
\hline Peniophora rufomarginata (Pers.) Litsch. & & . & . & . & &. & $\mathrm{x}^{2}$ & $2^{2}$ & &. \\
\hline Mycena polygramma (Bull.: Fr.) Gray & & & & & & & $21^{+-2}$ & $4^{1-2}$ & & . \\
\hline Mycena maculata P. Karst. & & & & & & & $18^{1-3}$ & $4^{2}$ & & \\
\hline Phlebia radiata Fr. & & & $\dot{\theta}$ & & & $\cdot$ & $14^{1-3}$ & $5^{1-2}$ & & . \\
\hline Crepidotus mollis (Schaeff.: F & & & & & & & $13^{1-2}$ & $5^{1-2}$ & & \\
\hline
\end{tabular}


Tab. 2. cont.

\begin{tabular}{|c|c|c|c|c|c|c|c|c|c|c|}
\hline Cyathus striatus (Huds.) Willd.: Pers. & & &. &. & $\dot{.}$ & & $9^{1-4}$ & $3^{2}$ & & \\
\hline Xerula radicata (Relh.: Fr.) Dörfelt & & & & & & & $8^{+-2}$ & & & \\
\hline Schizophyllum commune Fr.: Fr. & & & & . & & & $7^{2}$ & $4^{2}$ & & \\
\hline Pleurotus pulmonarius (Fr.) Quél. & & & &. & & & $7^{+-2}$ & $3^{1-2}$ & . & \\
\hline Psathyrella candolleana (Fr.: Fr.) Maire & & & & & & & $5^{2-3}$ & & & \\
\hline Polyporus varius (Pers.): Fr. & & & $\cdot$ & . & & & $5^{+-1}$ & $3^{+-1}$ & & \\
\hline Polyporus arcularius (Batsch): Fr. & & & $\dot{.}$ & $\cdot$ & & & $4^{+-1}$ & & & \\
\hline Encoelia furfuracea (Roth) P. Karst. & & & & & & & $3^{2}$ & & & \\
\hline Pluteus salicinus (Pers.: Fr.) P. Kumm. & & & & & & & $2^{+-1}$ & & & \\
\hline Tremella foliacea Pers. & & . &. & . & . & & $2^{1}$ & $2^{1}$ & . & \\
\hline Bisporella citrina (Batsch: Fr.) Korf \& S. E. Carp. & & & & . & & & $2^{2}$ & $5^{2-3}$ & & \\
\hline Clavariadelphus fistulosus (Holmsk.: Fr.) Coener & & & & & & & $2^{1}$ & & & \\
\hline Genoderma lucidum (M. A. Curtis: Fr.) P. Karst. & . & . & . & . & . & . & $2^{+}$ & & & \\
\hline Pleurotus ostreatus (Jacq.: Fr.) P. Kumm. & . & & . & . & & & $1^{1}$ & $2^{1-2}$ & & \\
\hline Lentinellus cochleatus (Pers.: Fr.) P. Karst. & & & & & & & $1^{2}$ & $1^{2}$ & & \\
\hline Crucibulum leave (Huds.) Kambly &. & . & . & . & . & . & $1^{1}$ & . & . &. \\
\hline Paxillus atrotomentosus (Batsch: Fr.) Fr. & & & & - & & & $1^{+}$ & & & \\
\hline Xerula pudens (Pers.) Singer & & & & & & & $1^{2}$ & & & \\
\hline Bjerkandera fumosa (Pers.: Fr.) P. Karst. & . & . & . & . & $\cdot$ & . & . & $8^{2}$ & . &. \\
\hline Panellus stypticus (Bull.: Fr.) P. Karst. & & & & 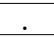 & & & & $6^{1-3}$ & & \\
\hline Calocera cornea (Batsch: Fr.) Fr. & & & . & & & & . & $4^{1-2}$ & & \\
\hline $\begin{array}{l}\text { Ascocoryne sarcoides (Jacq.) J. W. Groves \& D. E. } \\
\text { Wilson }\end{array}$ & . & . & . & . & • & . & . & $4^{2}$ & . & . \\
\hline Cerrena unicolor (Bull.: Fr.) Murrill & . & . & . & . & . & . & . & $4^{2}$ & . & . \\
\hline Flammulina velutipes (M. A. Curtis: Fr.) Singer & & & & - & & & & $3^{2-3}$ & & \\
\hline Bulgaria inquinalis (Pers.) Fr. & & & & & & &. & $2^{2}$ & & \\
\hline Lenzites betulinus (L.: Fr.) Fr. & . & & $\dot{ } \cdot$ & . & & & . & $2^{2}$ & & $\dot{\sigma}$ \\
\hline Coprinus micaceus (Bull.: Fr.) Fr. & . & . & . & . & & & . & $2^{3-4}$ & . & \\
\hline Pleurotus dryinus (Pers.: Fr.) P. Kumm. & & & & & & & 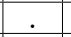 & $1^{+}$ & & \\
\hline Pycnoporus cinnabarinus (Jacq.: Fr.) P. Karst. & . & & . &. & & & . & $1^{1}$ & & \\
\hline Peniophora limitata (Chaillet: Fr.) Cooke & . & . & . & . & & . & . & . & $\mathrm{x}^{2}$ & $1^{2}$ \\
\hline $\begin{array}{l}\text { Chlorociboria aeruginosa (Pers.: Fr.) Seaver ex C. S. } \\
\text { Ramamuthri, Korf \& L. R. Batra }\end{array}$ & . & & . & . & . & . & . & . & $2^{+-1}$ & . \\
\hline Daedaleopsis confragosa (Bolt.: Fr.) J. Schröt. & & & &. & & & . &. & $\mathrm{x}^{1}$ & $1^{1}$ \\
\hline Stereum subtomentosum Pouzar & & & $\cdot$ & . & & & . &. & $\mathrm{x}^{2}$ & . \\
\hline Mycena niveipes Murrill & & & & & & & & & $4^{1-2}$ & \\
\hline Pluteus nanus (Pers.: Fr.) P. Kumm. & & & &. & & & . & . & $1^{+}$ & \\
\hline Hypoxylon fuscum (Pers.: Fr.) Fr. & & & & & & & & & & $1^{2}$ \\
\hline \multicolumn{11}{|c|}{ Bryophilous fungi } \\
\hline Number of species & & \multicolumn{2}{|c|}{5} & \multicolumn{2}{|c|}{5} & & \\
\hline Galerina hypnorum (Schrank: Fr.) Kühner & $2^{1}$ & $1^{1}$ & & & $27^{+-2}$ & $2^{1}$ & $11^{+-2}$ & $4^{+-1}$ & $10^{1-2}$ & \\
\hline Rickenella fibula (Bull.: Fr.) Raith. & $2^{1}$ & & & & $7^{1-2}$ & $1^{1}$ & $16^{+-2}$ & $2^{1-2}$ & $9^{+-3}$ & \\
\hline Mycena acicula (Schaeff.) P. Kumm. & & & & & $9^{+-1}$ & & $17^{+-2}$ & $2^{1}$ & & \\
\hline Galerina paludosa (Fr.) Kühner & & & $14^{+-2}$ & $2^{1-2}$ & & & & & & \\
\hline Galerina sphagnorum (Pers.: Fr.) Kühner & & & $13^{+-2}$ & & & & . & . & & \\
\hline Psilocybe elongata (Pers.: Fr.) J. E. Lange & & & $10^{+-2}$ & $1^{1}$ & & & & & & \\
\hline Lyophyllum palustre (Peck) Singer & & & $15^{+-2}$ & $1^{+}$ & & & & & & \\
\hline Omphalina sphagnicola (Berk.) M. M. Moser & & & 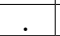 & $1^{+}$ & & & & & & \\
\hline Galerina mniophila (Lasch) Kühner & & & & 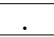 & $9^{+-1}$ & $2^{1}$ & & & & \\
\hline Leotia lubrica (Scop.) Pers. & & & & $\dot{ } \cdot$ & & $2^{1-2}$ & & & & \\
\hline Rickenella setipes (Fr.: Fr.) Raith. & & & & & & & $9^{+-1}$ & $2^{1}$ & $7^{+-1}$ & \\
\hline \multicolumn{11}{|c|}{ Parasitic fungi } \\
\hline Number of species & & & 3 & & 10 & & 11 & 1 & & \\
\hline Fomes fomentarius (L.: Fr.) Kickx & & & $\mathrm{x}^{1}$ & & & $2^{1}$ & $2 \mathrm{x}^{1}$ & $13^{1-2}$ & & $1^{1}$ \\
\hline Fomitopsis pinicola (Swartz: Fr.) P. Karst. & & & $29^{1}$ & $1^{+}$ & $\mathrm{x}^{1}$ & $2^{1}$ & $2 x^{1-2}$ & $1^{1}$ & & \\
\hline Sparassis crispa (Wulf.): Fr. & $2^{+}$ & & &. & $1^{+}$ & $2^{+-1}$ & $\dot{.}$ & $\dot{.}$ & & \\
\hline Heterobasidion annosum (Fr.) Bref. ss. lato & & $2^{+-1}$ & & & $\mathrm{x}^{1-2}$ & & & & & \\
\hline Inonotus obliquus (Pers.: Fr.) Pilát & & $1^{1}$ &. & $1^{+}$ & & & & & & \\
\hline Armillaria ostoyae (Romagn.) Herink & & & & -1 & $2^{1}$ & $2^{1-2}$ & $5^{2}$ & $4^{1-2}$ & $2^{1-2}$ & \\
\hline Laetiporus sulphureus (Bull.: Fr.) Murrill & & & & & & $5^{+-1}$ & $4^{1-2}$ & $2^{+-1}$ & & \\
\hline
\end{tabular}


Tab. 2. cont.

\begin{tabular}{|c|c|c|c|c|c|c|c|c|c|c|}
\hline Tremella encephala Pers.: Fr. & & . & . & . & $13^{+-2}$ & $2^{1}$ & . & . & . & \\
\hline Phellinus pini (Brot.: Fr.) A. Ames & & . & . & . & & $1^{1}$ & . & . & . & . \\
\hline Cordyceps capitata (Holmskj.: Fr.) Fr. & & & & & & $1^{2}$ & & & & \\
\hline Cordyceps ophioglossoides (Ehrh. ex Pers.: Fr.) Fr. & & • & & & & $1^{1}$ & & & & \\
\hline Pholiota squarrosa (Weigel: Fr.) P. Kumm. & & . & . & . & & . & $5^{1}$ & & $3^{2-4}$ & \\
\hline Dumontinia tuberosa (Hedw.) L. M. Kohn & & . & . & . & & . & $2^{2}$ & $1^{1}$ & $4^{1-2}$ & $1^{1}$ \\
\hline Inonotus radiatus (Sowerby: Fr.) P. Karst. & & - & & & & 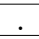 & & $1^{3}$ & & $1^{2}$ \\
\hline Fistulina hepatica (Schaeff.): Fr. & & . & & . & & . & $8^{+-1}$ & $2^{+-1}$ & & . \\
\hline Phellinus robustus (P. Karst.) Bourdot \& Galzin & & . & . & . & & . & $\mathrm{x}^{1}$ & $1^{1}$ & . & \\
\hline Phellinus alni (Bondartsev) Parmasto & & 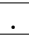 & . & . & . & . & $\cdot$ & $1^{1}$ & $\cdot$ & . \\
\hline Asterophora parasitica (Pers.: Fr.) Singer & . & . & . & . & & . & 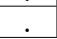 & $1^{2}$ & . & . \\
\hline
\end{tabular}

Abbreviations: P.-P. - Peucedano-Pinetum, V.u.-P. - Vaccinio uliginosi - Pinetum, S.-P. - Serratulo-Pinetum, T.-C. - Tilio-Carpinetum, Fr.-Al. - Fraxino-Alnetum; pp - permanent plots, cp - complementary plots; 1 , $2,3 \ldots$ - the number of records at a given plot; $\mathrm{x}$ - which corresponds to the sum of all observations at a specific plot, is used for species producing permanent fruit-bodies; ${ }^{+-5}-$ the commonly used Moser scale in the quantitative assessment.

Three hundred thirty five taxa of macromycetes were recorded in the phytocoenoses studied. A total of 354 macromycete species were observed between 1997 and 2000. The total number of taxa of macromycete species, including literature data, reported from the area is 506.

Peucedano-Pinetum (W. Mat. 1962) W. Mat. \& J. Mat. 1973

Mycological observations in Peucedano-Pinetum were conducted in one permanent plot (no. 1) and at 17 supplementary plots. A total of 58 observations in communities classified as Peucedano-Pinetum were performed. The occurrence of 73 macromycete species was recorded in the association (Tab. 2). Eleven macromycete species were exclusive of the association in the MLP, which is $15.3 \%$ of the mycobiota in Peucedano-Pinetum. Those are: Boletus pinophilus, Chalciporus piperatus, Cortinarius cinnamoneus, Gomphidius roseus, Hygrophorus hypothejus, Leccinum versipelle, L. vulpinum, Rozites caperatus, Suillus bovinus, Russula versicolor and Tricholoma equestre. The highest number of species in common was recorded between Peucedano-Pinetum and Serratulo-Pinetum (60 species, over $83 \%$ ).

Peucedano-Pinetum is an association characteristic of northeastern Poland. It is also known as Vaccinio myrtilli-Pinetum Kobendza 1930 (Matuszkiewicz 2001). Macromycetes occurring in Peucedano-Pinetum, mostly from the Białowieża Old Growth Forest - Project CRYPTO, are reported only sporadically in the available literature (Nespiak 1959; Chmiel, Sadowska 1994; Lisiewska 1995; Bujakiewicz 1995; Chmiel 1995, 1996; Skirgiełło 1995). Mycological observations in Peucedano-Pinetum outside the Białowieża Old Growth Forest have been conducted by, for instance, Lisiewska (1991-1992) and Kuszczyński (2007). Data on the mycobiota of Leucobryo-Pinetum, which is vicariant with Peucedano-Pinetum and which occurs in other parts of Poland, are reported in mycological literature more often (e.g., Lisiewska 1978; Lisiewska, Wójcik 1984; Friedrich 1985, 1994; Ławrynowicz, Szkodzik 1998; Łuszczyński 2007).

The mycobiota of the Peucedano-Pinetum phytocoenoses examined in the MLP corresponds to literature data; this is probably related to the characteristic species composition of Peucedano-Pinetum and its vicariant Leucobryo-Pinetum. Macromycete species associated with Pinus sylvestris dominate in both types of coniferous forests. 


\section{Vaccinio uliginosi-Pinetum Kleist 1929}

Observations in Vaccinio uliginosi-Pinetum were conducted in one permanent plot (no. 2) and 2 supplementary plots. A total of 41 observations were performed (Tab. 2). The occurrence of 35 macromycete species, including 13 (37.1\%) species exclusive of the community, was recorded in Vaccinio uliginosi-Pinetum. Species that occur only in Vaccinio uliginosi-Pinetum are as follows: Cortinarius huronensis, Galerina paludosa, G. sphagnorum, Lactarius helvus, L. thejogalus, Leccinum niveum, Lyophyllum palustre, Omphalina sphagnicola, Psilocybe elongata, Russula betularum, $R$. emetica var. emetica, R. paludosa and Rhodocollybia maculata.

The greatest resemblance of the mycobiota in the association was observed in Serratulo-Pinetum (19 species in common between both associations $-54.3 \%$ of the biota) and Peucedano-Pinetum (17 species in common between both associations $-48.6 \%$ of the biota). It is understandable given the coniferous character of the association and the dominance of Pinus sylvestris.

The mycobiota of Vaccinio uliginosi-Pinetum has been examined in a few mycocoenological studies (e.g. Nespiak 1959; Bujakiewicz 1986; Lisiewska 1978; Kałucka 1995; Friedrich 1997; Łuszczyński 2007). The majority of macromycete species recorded in the association have also occurred in patches of Vaccinio uliginosi-Pinetum in other parts of Poland. Those were, for instance, Cortinarius huronensis, Galerina paludosa, G. sphagnorum, Lactarius helvus, Leccinum niveum, Lyophyllum palustre, Psilocybe elongata, Russula emetica var. emetica, R. paludosa (Bujakiewicz 1986; Kałucka 1995).

Serratulo-Pinetum (W. Mat. 1981) J. Mat. 1988

Macromycetes in Serratulo-Pinetum were examined in 2 permanent plots (nos. 6 and 9) and 25 supplementary plots (Tab. 2). A total of 109 observations were performed in the association. The occurrence of 171 macromycete species was recorded. Fifty six species exclusive of the association were found $(32.4 \%$ of the mycobiota of the association). Those included: Agaricus silvaticus, Boletus edulis, Chroogomphus rutilus, Clitocybe clavipes, Lactarius deterrimus, Lycoperdon umbrinum, Macrolepiota rhacodes, Mycena zephirus, Russula mustelina, R. xerampelina, Stereum sanguinolentum or Strobilurus esculentus. The mixed tree-stand affects the number of species in common between Serratulo-Pinetum phytocoenoses and other plant associations. The greatest number of species in common was recorded in Tilio-Carpinetum - 76 species $(43.9 \%)$ and Peucedano-Pinetum - 60 species $(34.7 \%)$. This is characteristic of a community that exhibits features of both coniferous forests and oak-hornbeam forests and was observed in both permanent plots. The plot with a distinct participation of Picea abies (plot 6, tab. 1) exhibits more features of the coniferous forest, which corresponds to fungal species observed in the plot (e.g., Pseudohydnum gelatinosum, Ramaria abietina, Russula mustelina, Strobilurus esculentus, etc.). A considerable participation of Quercus robur in plot 9 results in its greater affiliation with broadleaved communities, which is reflected in the species composition of macromycetes (e.g., Cystolepiota seminuda, Tricholoma sulphureum, Hyphodontia paradoxa, etc.).

Similarly to Peucedano-Pinetum, the mycobiota of Serratulo-Pinetum is one of the most mycocoenologically under-explored mycobiotas in Poland. The subboreal mixed coniferous forest occurs in northeastern Poland and sporadically in central and eastern Poland. It is known as Pino-Quercetum serratuletosum or Calamagrostio 
arundinaceae-Piceetum in older studies from northeastern Poland (Matuszkiewicz 2001). Data on the mycobiota of Serratulo-Pinetum are available in studies by Nespiak (1959), Lisiewska (1991-1992) and Łuszczyński (2007). The mycobiota in the Serratulo-Pinetum phytocoenosis is intermediate between coniferous communities and oak-hornbeam communities. Species characteristic of both coniferous communities and broadleaved communities occurred there.

Tilio-Carpinetum Tracz. 1962

Macromycetes of oak-hornbeam forests represented by Tilio-Carpinetum were observed in 3 permanent plots (nos. 4, 8, 10; Tab. 1) and 23 supplementary plots in the MLP. A total of 169 observations were performed and a total of 198 macromycete species were recorded. No considerable differences in the species composition among individual permanent plots were observed. The mycobiota of Tilio-Carpinetum was characterised by the highest participation of exclusive species -78 ones, that is $39.4 \%$ of the mycobiota of the association. Clavulina coralloides, Cylindrobasidium laeve, Mycena maculata, Peniophora quercinum, P. rufomarginata, Phlebia radiata, Phellinus robustus, Xylaria longipes were the most frequently recorded species exclusive to oak-hornbeam forests in the MLP. Species exclusive of the oak-hornbeam forest also included: Amanita phalloides, Clavariadelphus fistulosus, Entoloma nidorosum, Fistulina hepatica, Leccinum pseudoscabrum, Macrolepiota procera, Pleurotus pulmonarius, Tricholoma lascivum, Russula risigallina, Xerula pudens.

The greatest similarity was observed between the mycobiota of the oak-hornbeam forest and fungi in Serratulo-Pinetum (76 species occurring in both; $38.4 \%$ of the mycobiota in the association). The number of species that occurred in TilioCarpinetum and other broadleaved communities was at a similar level: slightly over $20 \%$ of the mycobiota in the association.

The species composition of macromycetes in Tilio-Carpinetum has been studied intensely in Poland (e.g., Nespiak 1959; Lawrynowicz 1973; Lisiewska 1978; Gumińska 1991-1992; Skirgiełło 1998; Wojewoda et al. 1999; Ławrynowicz et al. 2002, Łuszczyński 2007). The mycobiota of the association examined in this study differentiates it well from other forest communities investigated in the MLP. The species composition of macromycetes in Tilio-Carpinetum is mostly consistent with fungal species occurring in oak-hornbeam phytocoenoses in other parts of Poland.

The analysis shows that the greatest similarity of the species composition and the number of species is observed between the local mycobiota of Tilio-Carpinetum in the MLP and the mycobiotas in Tilio-Carpinetum recorded in Central Poland (Ławrynowicz 1973) and the Skołczanka Reserve (Gumińska 1991-1992) and in the Tuchola Forests (Ławrynowicz et al. 2002).

Fraxino-Alnetum W. Mat. 1952

The mycobiota of Fraxino-Alnetum was examined in one permanent plot (no. 7; Tab. 1) and 2 supplementary plots. A total of 41 mycological observations were performed. 66 species of macromycetes were recorded (Tab. 2). Exclusive species (ten species) constituted $15.2 \%$ of the association mycobiota. Cortinarius paleaceus, Daedaleopsis confragosa, Lactarius lilacinus, Mutinus caninus, Mycena niveipes, $M$. rubromarginata, Paxillus rubicundulus, Pluteus nanus, Ramaria aurea and Stereum subtomentosum occurred only in Fraxino-Alnetum in the MLP. Fraxino-Alnetum and Tilio-Carpinetum had the greatest number of species in common (46 species, 69.7\% of the association biota). 


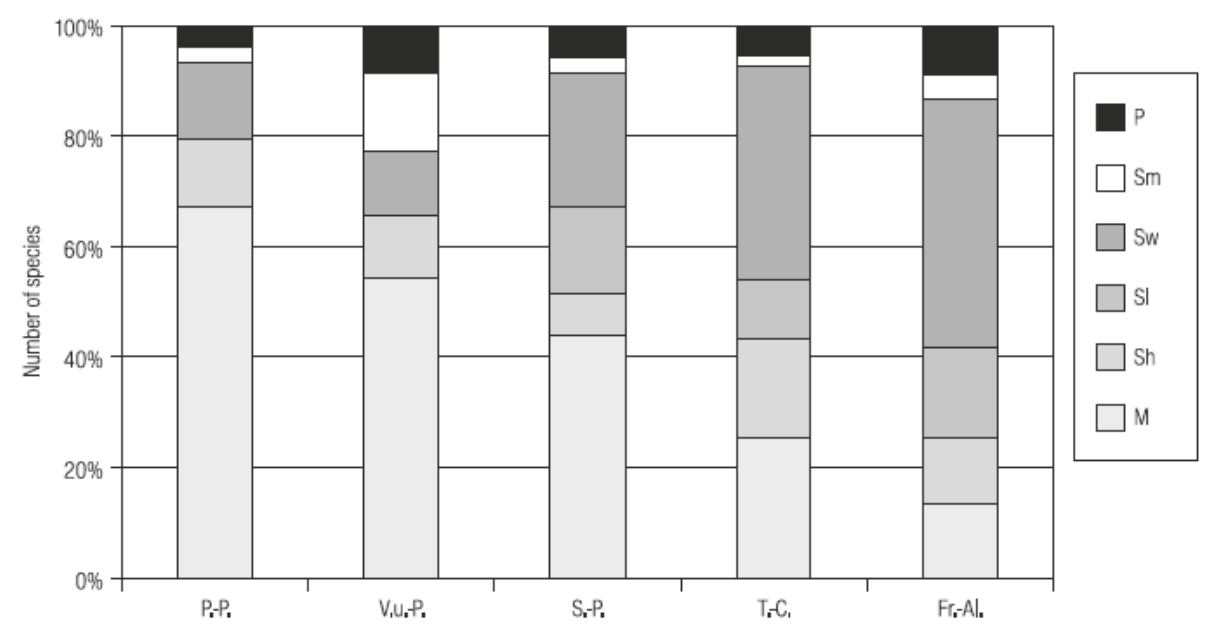

Fig. 2. The participation of bioecological groups of macrofungi in the forest communities of the Masurian Landscape Park.

P.-P. - Peucedano-Pinetum, Vu.-P. - Vaccinio uliginosi-Pinetum, S.-P. - Serratulo-Pinetum, T.-C. - TilioCarpinetum, Fr-Al. - Fraxino-Alnetum; M - mycorrhizal fungi, Sh - saprotrophic fungi on humus, S1 - saprotrophic fungi on litter, Sw - saprotrophic fungi on wood, Sm - saprotrophic fungi on mosses, $\mathrm{P}$ - parasitic fungi.

Macromycetes in Fraxino-Alnetum (=Circaeo-Alnetum) have been examined in numerous studies from almost entire Poland (e.g., Nespiak 1959; Bujakiewicz 1969, 1973, 1994; Lisiewska 1978; Friedrich 1985, 1994; Bujakiewicz, Fiebich 1991-1992; Euszczyński 2007). Species of mycorrhizal fungi characteristic of Alnus, Lactarius lilacinus and Cortinarius paleaceus (Friedrich 1994; Łuszczyński 2007), were recorded in the plot. Galerina hypnorum and Rickenella fibula were found on mossy trunk bases of alder trees (Euszczyński 2007). The species composition of macromycetes recorded in Fraxino-Alnetum was similar to that of analogous Fraxino-Alnetum forests in other parts of Poland. A smaller number of recorded species should be attributed to a limited surface area of permanent plots.

The analysis of participation of individual bioecological groups of macromycetes in the phytocoenoses examined here shows differences between mycorrhizal species and saprotrophic fungi on wood. The greatest participation of mycorrhizal fungi was recorded in Peucedano-Pinetum phytocoenoses (66.7\% of the mycobiota) and the smallest in Fraxino-Alnetum (12.1\% of the mycobiota) (Fig. 2). The opposite

Table 3

Number of macrofungi species in plant communities of the Masurian Landscape Park

\begin{tabular}{|c|c|c|c|c|}
\hline \multirow[t]{2}{*}{ Plant community } & \multirow{2}{*}{$\begin{array}{l}\text { Total number } \\
\text { of macrofungi }\end{array}$} & \multicolumn{2}{|c|}{ Number of species in research plots } & \multirow{2}{*}{$\begin{array}{l}\text { Macrofungi/ } \\
\text { plants ratio }\end{array}$} \\
\hline & & macrofungi & vascular plants & \\
\hline Peucedano-Pinetum & 72 & 47 & 19 & $2.47: 1$ \\
\hline $\begin{array}{l}\text { Vaccinio uliginosi- } \\
\text { Pinetum }\end{array}$ & 35 & 29 & 16 & $1.81: 1$ \\
\hline Serratulo-Pinetum & 173 & 119 & 41 & $2.90: 1$ \\
\hline Tilio-Carpinetum & 198 & 163 & 43 & $3.79: 1$ \\
\hline Fraxino-Alnetum & 66 & 52 & 26 & $2.00: 1$ \\
\hline
\end{tabular}


ratio was observed for saprotrophic fungi on wood which was only $11.4 \%$ for Vaccinio uliginosi-Pinetum and $13.9 \%$ for Peucedano-Pinetum in coniferous associations. Their greatest values were observed for the Fraxino-Alnetum phytocoenosis $(45.4 \%$ for Fraxino-Alnetum).

The ratio between the number of macromycete species and the number of plants in permanent plots in the phytocoenoses examined ranges from 1:1.81 for Vaccinio uliginosi-Pinetum to 3.79:1 for Tilio-Carpinetum (Tab. 3). The data are comparable with the results obtained by Łuszczyński (2007). However, the ratio is 1:1.81 (506 macromycete species : 920 plant species) for the total number of macromycete species recorded in the MLP (the author's observations and literature data) and the number of plant species (Kruszelnicki 1996). However, the observations by Mułenko (1998) and Grzywacz (1999), revealed that the number of fungal species is much greater than the number of plant species when macromycetes are also included.

\section{SUMMARY}

Three hundred and thirty five taxa of macromycetes were recorded in the phytocoenoses examined in the Masurian Landscape Park; 354 macromycete species were observed during examinations conducted in the Park between 1997 and 2000. The total number of species reported from the Masurian Landscape Park, including literature data, is 506 .

Tilio-Carpinetum (198 taxa) and Serratulo-Pinetum (171 taxa) were the richest phytocoenoses in macromycete species. This is confirmed by the relationship between the number of macromycete species occurring in them and the diversified tree-stand (the diversity of substrate available for mycelium development).

Seventy three taxa of macromycetes were recorded in Peucedano-Pinetum. The association in the study area is differentiated by a group of exclusive species $(15.5 \%$ of the mycobiota of the association) such as Boletus pinophilus, Cortinarius cinnamoneus, Rozites caperatus, Russula versicolor or Tricholoma equestre as well as a group of species that it has in common with the Serratulo-Pinetum phytocoenosis ( $83 \%$ of the mycobiota of the association).

The species composition of macromycetes in Serratulo-Pinetum is characterised by a high participation of species in common with broadleaved communities $(47.9 \%$ of the mycobiota in common with Tilio-Carpinetum) and with coniferous forests (34.7\% of the mycobiota in common with Peucedano-Pinetum). $32.4 \%$ of exclusive species was recorded in the continental mixed coniferous forest.

A downward trend in the percentage participation of mycorrhizal species from coniferous communities (43-66\% of the mycobiota of the associations) to broadleaved communities (12-27\% of the mycobiota) was observed. A reverse trend was noted in the case of saprotrophic fungi on wood. The greatest participation of this group was recorded in Tilio-Carpinetum (ca 35\%) and in Fraxino-Alentum (45\%), whereas the smallest participation was observed in coniferous forests (11-23\%). This probably results from greater diversification of the tree-stand and the richness of available substrate. 


\section{REFERENCES}

Abromeit J. 1905. Bericht über die 43 Jahresversammlung in Culm am 7. October 1904. Schr. physik.ökon. Ges. Königsberg 46: 50-83.

Bajkiewicz-Grabowska E. 1989. Charakterystyka geograficzna i klimatyczna Mazurskiego Parku Krajobrazowego oraz podział hydrograficzny na wydzielone zlewnie jeziorne. (In:) A. Hillbricht-Ilkowska (ed.). Jeziora Mazurskiego Parku Krajobrazowego - stan eutrofizacji, kierunki ochrony. Zeszyty Naukowe Komitetu „Człowiek i Środowisko” PAN, 1: 13-20.

Bujakiewicz A. 1969. Udział grzybów wyższych w lasach łęgowych i olesach Puszczy Bukowej pod Szczecinem. Bad. Fizjogr. Pol. Zach., B 23: 61-96.

Bujakiewicz A. 1973. Udział grzybów wyższych w lasach łęgowych i w olesach Wielkopolski. PTPN, Prace Komisji Biol. 25 (6): 1-92.

Bujakiewicz A. 1986. Udział macromycetes w zbiorowiskach roślinnych występujących na podłożu torfowym w Słowińskim Parku Narodowym. Bad. Fizjogr. Pol. Zach., B 37: 101-129.

Bujakiewicz A. 1994. Macrofungi in the alder forests of the Białowieża National Park. Mycologia Helvetica 6 (2): 57-76.

Bujakiewicz A. 1995. Fungi. Agaricales. (In:) J. B. Faliński, W. Mułenko (eds). Cryptogamous plants in the forest communities of Białowieża National Park. General problems and taxonomic groups analysis. (Project CRYPTO 2). Phytocoenosis 7 (N.S.), Archiv. Geobot. 4: 141-148.

Bujakiewicz A., Fiebich R. 1991-1992. Udział ekologicznych grup macromycetes w płatach olsu w Wielkopolskim Parku Narodowym. Acta Mycol. 27 (1): 63-91.

Chmiel M. A. 1995. Discomycetous fungi. (In:) J. B. Faliński, W. Mułenko (eds). Cryptogamous plants in the forest communities of Białowieża National Park. General problems and taxonomic groups analysis. (Project CRYPTO 2). Phytocoenosis 7 (N.S.), Archiv. Geobot. 4: 115-123.

Chmiel M. A. 1996. Litter saprobic fungi. (In:) J. B. Faliński, W. Mułenko (eds). Cryptogamous plants in the forest communities of Białowieża National Park. Functional groups analysis and general synthesis. (Project CRYPTO 3). Phytocoenosis 8 (N.S.), Archiv. Geobot. 6: 111-117.

Chmiel M. 2006. Checklist of Polish larger Ascomycetes. (In:) Z. Mirek (ed.). Biodiversity of Poland. 8. W. Szafer Institute of Botany, Polish Academy of Sciences, Kraków, 152 pp.

Chmiel M. A., Sadowska B. 1994. Grzyby koprofilne w zbiorowiskach leśnych Białowieskiego Parku Narodowego. Fragm. Flor. Geobot. Ser. Polonica 1: 107-131.

Dą̧rowski St., Polakowski B., Wołos L. 1999. Obszary chronione i pomniki przyrody województwa warmińsko-mazurskiego. Urząd Wojewódzki, Wydział Ochrony Środowiska i Rolnictwa, Olsztyn, 130 pp.

Domański S. 1963. Fungi lignicoli in regione Mazury in Polonia septentrionali annis 1956-1961 collecti. Monogr. Bot. 15: 295-323.

Durska B. 1971. O występowaniu w Polsce Mycena belliae (Johnst.) Orton. Acta Mycol. 7 (1): 141-144.

Friedrich S. 1985. Macromycetes na tle zespołów leśnych Puszczy Goleniowskiej. Acta Mycol. 21 (1): 43-76.

Friedrich S. 1994. Charakterystyka socjologiczno-ekologiczna mikoflory zbiorowisk leśnych Cedyńskiego Parku Krajobrazowego. Rozprawy AR Szczecin, 161: 1-100.

Friedrich S. 1997. Macromycetes of the proposed nature reserve Wilcze Uroczysko-Olszanka in the Odra estuary. Acta Mycol. 32 (2): 239-255.

Friedrich S. 2000. Ocena metody zastosowanej w badaniach mikologicznych zbiorowisk leśnych Cedyńskiego Parku Krajobrazowego. Folia Univ. Agric. Stetin. 213 Agricultura (85): 95-104.

Grzywacz A. 1999. Gatunkowa różnorodność biologiczna grzybów. Zeszyty Nauk. AR im. H. Kołłątaja w Krakowie 348: 23-38.

Gumińska B. 1991-1992. Higher fungi of the Tilio-Carpinetum forest association in the Skołczanka reserve near Cracow. Acta Mycol. 27 (1): 137-158.

Hansen L., Knudsen H. (eds). 2000. Nordic macromycetes. 1. Ascomycetes. Nordsvamp, Copenhagen, $309 \mathrm{pp}$.

Kałucka I. 1995. Macromycetes in the forest communities of the Jodły Laskie nature reserve (Central Poland). Acta Mycol. 30 (1): 3-26.

Kondracki J. 1998. Geografia regionalna Polski. PWN, Warszawa, 440 pp.

Kotlaba F., Lazebniček J. 1967. IV Sjezd evropských mykologů, Polska 1966. Česká Mycol. 21 (1): 54-59.

Kruszelnicki J. 1996. Ekologiczne podstawy ochrony i kształtowania przyrody Mazurskiego Parku Krajobrazowego. Praca doktorska. IB PAN, Kraków (msc.). 
Ławrynowicz M. 1973. Grzyby wyższe makroskopowe w grądach Polski Środkowej. Acta Mycol. 9 (2): 133-204.

Lawrynowicz M., Szkodzik J. 1998. Macromycetes of the Kręgi Kamienne nature-archaeological reserve in the Bory Tucholskie (NW Poland). Acta Mycol. 33 (2): 327-340.

Lawrynowicz M., Dziedziński T., Szkodzik J. 2002. Macromycetes of Aceri-Tilietum and Tilio-Carpinetum in the „Dolina Rzeki Brdy” nature reserve in the Bory Tucholskie (NW Poland). Acta Mycol. 37 (1/2): 63-76.

Lisiewska M. 1965. Udział grzybów wyższych w grądach Wielkopolski. Acta Mycol. 1: 169-269.

Lisiewska M. 1978. Macromycetes na tle zespołów leśnych Świętokrzyskiego Parku Narodowego. Acta Mycol. 14 (1/2): 163-191.

Lisiewska M. 1991-1992. Flora macromycetes rezerwatu „Perkuć” w Puszczy Augustowskiej. Acta Mycol. 27 (2): 175-187.

Lisiewska M. 1995. Tremelloid fungi, Boletales, Elaphomycetales. (In:) J. B. Faliński, W. Mułenko (eds). Cryptogamous plants in the forest communities of Białowieża National Park. General problems and taxonomic groups analysis.(Project CRYPTO 2). Phytocoenosis 7 (N.S.), Archiv. Geobot. 4: 129-131, $137-140,153$.

Lisiewska M. 2000. Udział bioekologicznych grup macromycetes w zbiorowiskach acidofilnych dąbrów na Płycie Krotoszyńskiej w południowej Wielkopolsce. (In:) M. Lisiewska, M. Lawrynowicz (eds). Monitoring grzybów. PTB, Sekcja Mikologiczna, Poznań-Łódź: 27-51.

Lisiewska M., Wójcik J. 1984. Obserwacje mikosocjologiczne w niektórych zbiorowiskach leśnych okolic Kazimierza Biskupiego (województwo konińskie). Bad. Fizjogr. Pol. Zach., B 35: 25-34.

Luszczyński J. 2007. Diversity of Basidiomycetes in various ecosystems of the Góry Świętokrzyskie Mts. Monogr. Bot. 97: 1-218.

Matuszkiewicz J. M. 2001. Zespoły leśne Polski. PWN, Warszawa, 357 pp.

Mirek Z., Piękoś-Mirkowa H., Zając A., Zając M. 2002. Flowering plants and pteridophytes of Poland, a checklist. (In:) Z. Mirek (ed.). Biodiversity of Poland. 1. W. Szafer Institute of Botany, Polish Academy of Sciences, Kraków, 442 pp.

Mułenko W. 1998. Mikroskopijne grzyby fitopatogeniczne w strukturze naturalnych zbiorowisk leśnych. Rozprawy habilitacyjne LXII. Wyd. UMCS, Lublin, 188 pp.

Nespiak A. 1959. Studia nad udziałem grzybów kapeluszowych w zespołach leśnych na terenie Białowieskiego Parku Narodowego. Monogr. Bot. 8: 3-141.

Neuhoff W. 1933. Die Hymenomyceten Ostpreussens. Eine systematische Zusammenstellung. Unser Ostland 2 (7): 317-397.

Ochyra R., Żarnowiec J., Bednarek-Ochyra H. 2003. Census catalogue of Polish Moses. Biodiversity of Poland. 3. Szafer Institute of Botany, Polish Academy of Sciences, Kraków, 372 pp.

Olesiński L., Wojewoda W. 1985. Materiały do flory macromycetes północno-wschodniej Polski. Acta Mycol. 21 (2): 193-232.

Orłoś H., Dominik T. 1960. Z biologii huby korzeniowej - Fomes annosus (Fr.) Cooke. Sylwan 104: 1-13.

Polakowski B., Chudyba H., Dąbek E., Dziedzic J., Jutrzenka-Trzebiatowski A., Korniak T., Pietraszewski W. 1976. Zarys stosunków geobotanicznych Mazurskiego Parku Krajobrazowego. I. Zespoły roślinne parku. Zesz. Nauk. ART. Olszt., Rolnictwo 18: 31-40.

Polakowski B., Jutrzenka-Trzebiatowski A., Hołdyński Cz. 1997. Roślinność rzeczywista Mazurskiego Parku Krajobrazowego. Wydawnictwo ART. Olsztyn, ss. 40 + mapa 1:30 000.

Skirgiełło A. 1968. Compte-rendu du IV-eme Congres des Mycologues Européens. Warszawa 1966. Acta Mycol. 4 (2): 243-249.

Skirgiełło A. 1995. Gasteromycetous fungi, Aphyllophorales, Russulales, Mucorales. (In:) J. B. Faliński, W. Mułenko (eds). Cryptogamous plants in the forest communities of Białowieża National Park. General problems and taxonomic groups analysis. (Project CRYPTO 2). Phytocoenosis 7 (N.S.), Archiv. Geobot. 4: 125-128, 133-136, 149-151, 155-157.

Skirgiełło A. 1998. Macromycetes of oak-hornbeam forests in the Białowieża National Park - monitoring studies. Acta Mycol. 33 (2): 171-189.

Wojewoda W. 2003. Checklist of Polish larger Basidiomycetes. (In:) Z. Mirek (ed.). Biodiversity of Poland. 7. W. Szafer Institute of Botany, Polish Academy of Sciences, Kraków, 812 pp.

Wojewoda W., Heinrich Z., Komorowska H. 1999. Macromycetes of oak-lime-hornbeam woods in the Niepołomnice Forest near Kraków (S Poland) - monitoring studies. Acta Mycol. 34 (2): 201-266. 


\section{Udział macromycetes w wybranych zbiorowiskach leśnych Mazurskiego Parku Krajobrazowergo}

\section{Streszczenie}

W latach 1997-2000 na terenie Mazurskiego Parku Krajobrazowego prowadzono badania mikosocjologiczne, dotyczące występowania grzybów wielkoowocnikowych (macromycetes). W niniejszej pracy przedstawiono charakterystykę mikosocjologiczną 5, wybranych zespołów leśnych: Peucedano-Pinetum, Vaccinio uliginosi-Pinetum, Serratulo-Pinetum, Tilio-Carpinetum oraz Fraxino-Alnetum. Zastosowano ogólnie przyjętą metodę prowadzania badań mikosocjologicznych. Wielkość pojedynczej powierzchni wynosiła $400 \mathrm{~m}^{2}$.

W prezentowanych fitocenozach stwierdzono występowanie 335 taksonów macromycetes. Na terenie Parku, w trakcie badań własnych, odnotowano łącznie 354 taksony macromycetes, co z danymi z literatury daje łącznie 506 taksonów, których występowanie stwierdzono na obszarze Mazurskiego Parku Krajobrazowego.

W trakcie obserwacji mikologicznych określono skład gatunkowy badanych zespołów leśnych Parku. Najbogatsze w gatunki macromycetes okazały się fitocenozy Tilio-Carpinetum (198 taksonów) oraz Serratulo-Pinetum (171 taksony). Potwierdza to związek liczby występujących gatunków grzybów wielkoowocnikowych z bogatym drzewostanem - różnorodność dostępnego substratu dla rozwoju grzybni.

Prześledzono udział grzybów z poszczególnych grup bioekologicznych w badanych zbiorowiskach leśnych. Gatunki mikoryzowe stanowią od 43 do $66 \%$ mikobioty badanych zbiorowisk borowych. W zespołach lasowych udział ich sięga niespełna $27 \%$ mikobioty badanych fitocenoz. Odwrotna tendencja zaznaczyła się w przypadku saprotrofów nadrewnowych. Największy udział grupa ta miała w grądach (ok. 35\%) i łęgach (45\%), najmniejszy w borach (11-23\%). Potwierdza to związek liczby gatunków nadrewnowych z bogactwem dostępnego substratu (zróżnicowany drzewostan). 